\title{
Synthesis and antimicrobial activity of some 2- Piperidinomethylamino-4-(7-H/substituted coumarin-3-yl)- 6-chlorosubstitutedphenyl pyrimidines
}

\author{
Mohd Imran ${ }^{1 *}$, Ozair Alam ${ }^{2}$ and Abida ${ }^{1}$ \\ ${ }^{1}$ Department of Pharmaceutical Chemistry, Faculty of Pharmacy, Northern Border University, Rafha, 91911, PO Box 840, \\ Kingdom of Saudi Arabia, ${ }^{2}$ Department of Pharmaceutical Chemistry, Faculty of Pharmacy, Jamia Hamdard (Hamdard \\ University), New Delhi-110062, India
}

*For correspondence: Email: imran_inderlok@yahoo.co.in; Tel: +966535129629

\begin{abstract}
Purpose: To prepare and evaluate some 2-piperidinomethylamino-4-(7-H/substitutedcoumarin-3-yl)-6chlorosubstitutedphenyl pyrimidines as antimicrobial agents.

Methods: Some 2-piperidinomethylamino-4-(7-H/substitutedcoumarin-3-yl)-6-chlorosubstitutedphenyl pyrimidines were prepared by reacting 2-amino-4-(7-H/substitutedcoumarin-3-yl)-6(chlorosubstitutedphenyl) pyrimidines with piperidine and formaldehyde. The chemical structures of the synthesized compounds were elucidated by Fourier transform infrared (FTIR), ${ }^{1} \mathrm{H}$-nuclear magnetic resonance $\left({ }^{1} \mathrm{H}-\mathrm{NMR}\right)$, mass spectrometry and elemental analysis. These compounds were investigated for their antimicrobial activity against ten bacteria and five fungi by serial plate dilution method using standard drugs, namely, ofloxacin and ketoconazole, respectively, and their minimum inhibitory concentrations (MICs) were also determined.

Results: A total of eighteen new compounds (1a-18a) were synthesized. Compound 6 a $(M I C=50$ $\mu \mathrm{g} / \mathrm{mL} ; p<0.05$ or less) displayed the highest activity against $S$. aureus, E. faecalis, S. epidermidis, $B$. subtilis, and $B$. cereus. Compound 6a further showed good activity (MIC $=25 \mu \mathrm{g} / \mathrm{mL} ; p<0.05$ or less) against $E$. coli; $P$. aeruginosa K. pneumonia, B. bronchiseptica, and $P$. vulgaris. Compounds $6 \mathbf{a}$ (MIC = $25 \mu \mathrm{g} / \mathrm{mL} ; p<0.0001)$ and 17a $(M I C=25 \mu \mathrm{g} / \mathrm{mL} ; p<0.0001)$ displayed very good activity against $C$. albicans, $A$. niger, A. flavus, $M$. purpureous, and $P$. citrinum, respectively. Analysis of structure-activity relationship revealed that the presence of bromo group at 7-postion of the coumarin moiety along with the 4-chlorophenyl group at position-6 of the pyrimidine ring is critical for antimicrobial activity against Gram-positive bacteria, Gram negative bacteria and fungi.

Conclusion: The synthesized 2-piperidino derivatives are better antifungal and antibacterial agents than the earlier reported 2-morpholino derivatives, but require further investigations against other microbial strains to ascertain their broad spectrum antimicrobial activity.
\end{abstract}

Keywords: Pyrimidine, Coumarin, Piperidine, Antibacterial, Antifungal, Structure-activity relationship

Tropical Journal of Pharmaceutical Research is indexed by Science Citation Index (SciSearch), Scopus, International Pharmaceutical Abstract, Chemical Abstracts, Embase, Index Copernicus, EBSCO, African Index Medicus, JournalSeek, Journal Citation Reports/Science Edition, Directory of Open Access Journals (DOAJ), African Journal Online, Bioline International, Open-J-Gate and Pharmacy Abstracts

\section{INTRODUCTION}

Microbial infections have been creating problems for mankind since centuries and scientists have also developed a large number of antimicrobial agents for the treatment of these infections. According to one new report, about 40 new microbial diseases have been identified since 1970s and more than 2 million Americans are suffering from antibiotic resistance, of which 
about 23000 die each year [1]. Because of the development of antibiotic resistance and emergence of new microbial diseases, there is a need to develop new antimicrobial agents for the treatment of microbial infections.

Pyrimidine derivatives have an important place in medicinal chemistry as these are associated with a broad range of biological activities [2-7] including antimicrobial activity [8-13]. The clinical importance of pyrimidine nucleus is also evident by the marketing of clinically used pyrimidine derivatives as well as fused pyrimidine derivatives; for example, as antineoplastic agent (tegafur), as vasodilator (dipyridamole), as expectorant (tasuldine) and as antibacterial agent (trimethoprim, piromidic acid, tetroxoprim, metioprim), as antifungal agent (flucytosine), and as antiviral agent (broxuridine, idoxuridine) [14]. Recently, the significance and biological importance of pyrimidine derivatives including their clinical applications in the microbial world has been reviewed [15]. The antimicrobial activity of pyrimidine derivatives against broad range of microbes makes it an important skeleton in medicinal chemistry and drug development against microbes. The piperidine nucleus is also an important moiety in medicinal chemistry research [16]. A number of piperidine ring containing chemical compounds have also been reported as antimicrobial agents [17-20].

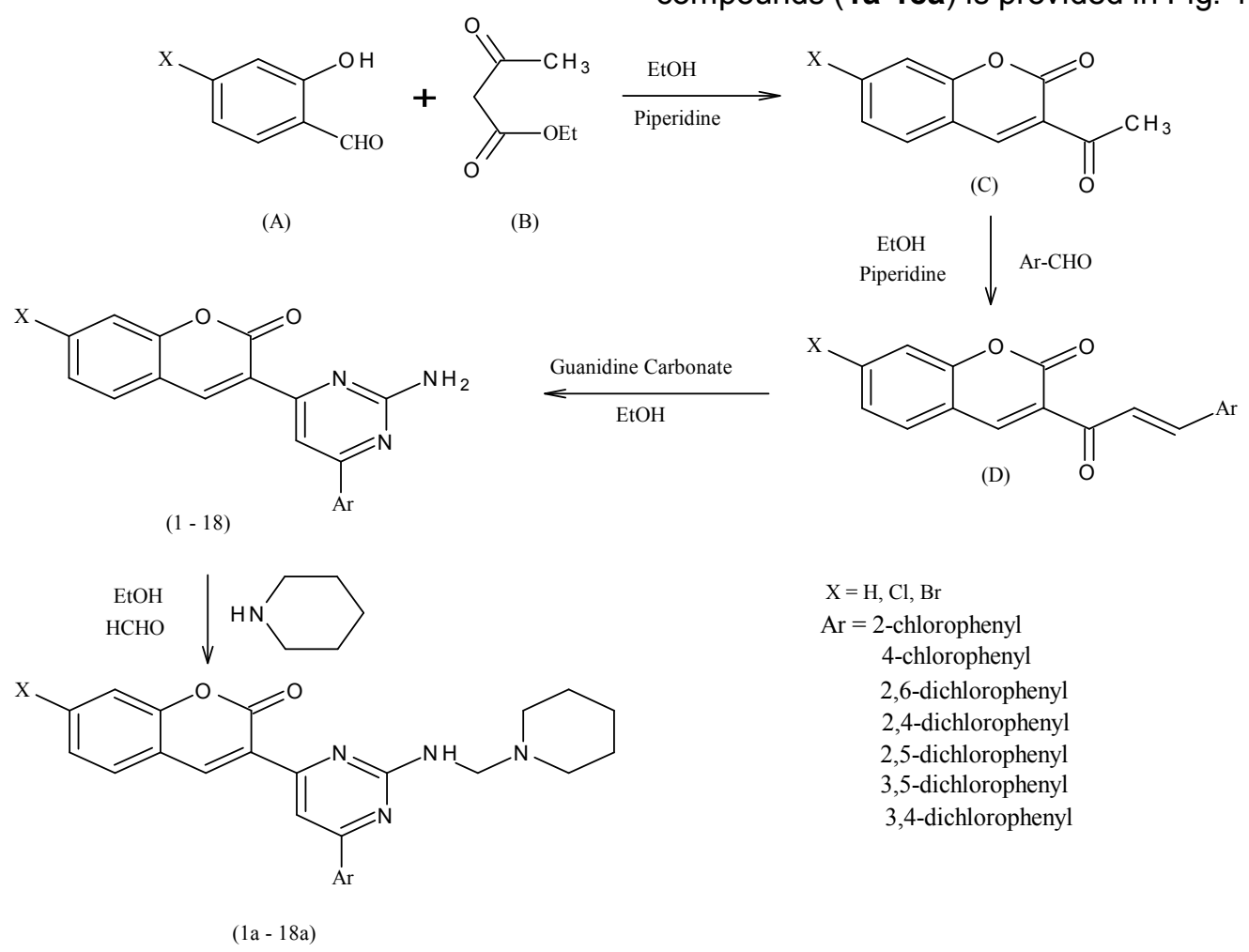

Encouraged by these observations and also in continuation of our search for potent antimicrobial agents [21,22] including antimicrobial agents having coumarin moiety [2325], we decided to prepare some 2piperidinomethylamino-4-(7-H/substitutedcoumarin-3-yl)-6-chlorosubstitutedphenyl pyrimidines, herein after the title compounds (1a-18a), as antimicrobial agents.

\section{EXPERIMENTAL}

\section{General}

Melting points were measured in open capillary tubes and are uncorrected. IR $(\mathrm{KBr})$ spectra were recorded on a Nicolet, 5PC FT-IR spectrometer (Browser Morner, USA) and 1H-NMR/13C-NMR spectra on a Bruker DRX-300 FT NMR (Bruker, Germany) spectrophotometer using TMS as internal reference (chemical shift in $\delta \mathrm{ppm}$ ). Mass spectra were recorded on a Jeol-JMS-D-300 mass spectrometer $(70 \mathrm{eV})$ (Jeol, Japan). Satisfactory analysis for $\mathrm{C}, \mathrm{H}$, and $\mathrm{N}$ was obtained for the compounds within $\pm 0.4 \%$ of the theoretical values. Purity of the compounds was checked on silica gel $G$ plates using iodine vapours as visualizing agent. $\mathrm{Rf}$ value of the compounds was determined by using a mixture of benzene and acetone (9:1). All reagents used in the present work were of analytical grade. The synthetic pathway for the preparation of the title compounds (1a-18a) is provided in Fig. 1.$$
\text { (1) }
$$

Figure 1: Synthesis of the title compounds $(1 \mathrm{a}-\mathbf{1 8 a})$ 
The 2-amino-4-(7-H/substitutedcoumarin-3-yl)-6(chlorosubstitutedphenyl) pyrimidines (1-18) prepared according to our previous report [23] were reacted with piperidine and formaldehyde in absolute ethanol to provide the title compounds (1a-18a).

\section{General method for the synthesis (1a-18a)}

A mixture of 2-amino-4-(7H/substitutedcoumarin-3-yl)-6-chlorosubstituted phenyl pyrimidines $(0.01$ mole), piperidine $(0.01$ mole) and formaldehyde (0.015 moles) was refluxed in absolute ethanol for 8 to $12 \mathrm{~h}$. The reaction mixture was reduced to half of its volume and poured on crushed ice. The solid separated was filtered, washed with water repeatedly, dried and recrystallized from ethanol.

\section{Evaluation of antimicrobial activity}

The title compounds (1a-18a) were tested for their in vitro antimicrobial activity by serial plate dilution method [26,27] against Gram-positive bacteria, Staphylococcus aureus (ATCC 25923), Enterococcus faecalis (ATCC 29212), Staphylococcus epidermidis (ATCC 12228), Bacillus subtilis (ATCC 6633) and Bacillus cereus (ATCC 9946); Gram-negative bacteria, Escherichia coli (ATCC 25922), Pseudomonas aeruginosa (ATCC 27853), Klebsiella pneumoniae (ATCC 700603), Bordetella bronchiseptica (ATCC 4617) and Proteus vulgaris (ATCC 9920); fungi, Candida albicans (ATCC 2091), Aspergillus niger (MTCC 281), Aspergillus flavus (MTCC 277), Monascus purpureous (MTCC 369) and Penicillium citrinum (NCIM 768). The microorganisms were obtained from the Institute of Genomics and Integrative Biology, New Delhi, India. Nutrient agar medium and Sabouraud dextrose medium were used for antibacterial activity and antifungal activity, respectively. The compounds were tested at concentrations of $200,175,150,125,100,75$, 50,25 and $12.5 \mu \mathrm{g} / \mathrm{mL}$. The reference or standard antibiotics, ofloxacin and ketoconazole were used at 50,25 and $12.5 \mu \mathrm{g} / \mathrm{mL}$ concentrations for antibacterial activity and antifungal activity, respectively. Sterile dimethyl sulfoxide (DMSO) was used for the preparation of desired concentrations of the synthesized compounds and standard antibiotics. Sterile dimethyl sulfoxide without the synthesized compounds and standard antibiotics served as control group. The minimum inhibitory concentrations (MICs) values of the synthesized compounds, ofloxacin and ketoconazole were also determined. The minimum inhibitory concentration (MIC) has been defined as the lowest concentration of a compound that inhibited visible growth of microorganisms on the plate.

\section{Statistical analysis}

All antimicrobial activity data are presented as mean \pm standard error of the mean (SEM, $n=6$ ). The data were analyzed by one-way analysis of variance (ANOVA) with Dunnett's multiple comparison test with respect to control and standard groups using GraphPad Prism version 5.00 for Windows (GraphPad Software, San Diego California USA). The results were considered significantly different at $p<0.05$.

\section{RESULTS}

The title compounds (1a-18a) were successfully prepared according to the method outlined in Fig. 1. The characterization data of the intermediates, (C), (D) and (1-18) of the Fig 1 were in line with our previously published data $[23,24]$. The structures of the title compounds were confirmed on the basis of their IR, ${ }^{1} \mathrm{H}-\mathrm{NMR},{ }^{13} \mathrm{C}-\mathrm{NMR}$, Mass and elemental analysis data. The appearance of the IR absorption peaks from 3277 to $3288 \mathrm{~cm}^{-1}$ confirmed the stretching vibration of $\mathrm{N}-\mathrm{H}$ group of $-\mathrm{NH}-\mathrm{CH}_{2^{-}}$moiety; from 1708 to $1712 \mathrm{~cm}^{-1}$ confirmed the stretching vibration of $\mathrm{C}=\mathrm{O}$ group of the coumarin moiety; from 1603 to $1611 \mathrm{~cm}^{-1}$ confirmed the stretching vibration of $\mathrm{C}=\mathrm{N}$ group of the pyrimidine ring; from 1540 to $1545 \mathrm{~cm}^{-1}$ confirmed the stretching vibration of $\mathrm{C}=\mathrm{C}$ group of aromatic $\mathrm{C}=\mathrm{C}$ bond; and from 1130 to 1133 $\mathrm{cm}^{-1}$ confirmed the stretching vibration of $\mathrm{C}-\mathrm{O}-\mathrm{C}$ group of coumarin moiety present in the title compounds (1a-18a). The appearance of the signals in the ${ }^{1} \mathrm{H}-\mathrm{NMR}$ spectra of the title compounds (1a-18a) at $\delta$ (ppm) values from 1.40 to 1.41 confirmed $2 \mathrm{H}$ of $\mathrm{C}-4$ of piperidine ring; from 1.55 to 1.57 confirmed the $4 \mathrm{H}$ of $\mathrm{C}-3$ and $\mathrm{C}$ 5 of piperidine ring; from 2.65 to 2.67 confirmed the $4 \mathrm{H}$ of $\mathrm{C}-2$ and $\mathrm{C}-6$ of piperidine ring; from 4.23 to 4.25 confirmed two methylene protons of $-\mathrm{NH}-\mathrm{CH}_{2}-$ moiety; from 6.91 to 7.70 confirmed the number of aromatic protons; and from 7.80 to 7.86 confirmed the secondary amino group (exchangable with $\mathrm{D}_{2} \mathrm{O}$ ) of $-\mathrm{NH}-\mathrm{CH}_{2}-$ moiety of the title compounds (1a-18a). The appearance of the signals in the ${ }^{13} \mathrm{C}-\mathrm{NMR}$ spectra of the title compounds (1a-18a) at $\delta$ (ppm) values from 26.3 to 26.5 confirmed the $\mathrm{C}-4$ of piperidine ring; from 27.4 to 27.5 confirmed the C-3 \& C-5 of piperidine ring; from 53.4 to 53.5 confirmed the C-2 \& C-6 of piperidine; and from 72.3 to 72.5 confirmed the amino methylene carbon of $-\mathrm{NH}-$ 
$\mathrm{CH}_{2-}$ moiety. Other ${ }^{13} \mathrm{C}-\mathrm{NNR}$ peaks, elemental analysis data and molecular ion peaks of the title compounds (1a-18a) were also consistent with the assigned structures.

The detailed physical constants, FTIR, ${ }^{1} \mathrm{H}-\mathrm{NMR}$, ${ }^{13} \mathrm{C}-\mathrm{NMR}$, Mass and elemental analysis data of the title compounds (1a-18a) are presented as follows.

\section{2-(Piperidinomethylamino)-4-(coumarin-3-yl)- 6-(4-chlorophenyl) pyrimidine (1a)}

Yield: 65 \%; m.p.: $165-167{ }^{\circ} \mathrm{C}$; $\mathrm{R}_{\mathrm{f}}$ : 0.69; IR (KBr) $\mathrm{cm}^{-1}: 3277(\mathrm{~N}-\mathrm{H}), 1708$ (C=O), $1609(\mathrm{C}=\mathrm{N}), 1541$ $(\mathrm{C}=\mathrm{C}), 1130$ (C-O-C); ${ }^{1} \mathrm{H}-\mathrm{NMR}\left(\mathrm{CDCl}_{3}\right.$, DMSO$\left.\mathrm{d}_{6}\right) \delta$ ppm: $1.40(\mathrm{~m}, 2 \mathrm{H}$ of $\mathrm{C}-4$ of piperidine $), 1.57$ ( $\mathrm{m}, 4 \mathrm{H}$ of $\mathrm{C}-3$ and $\mathrm{C}-5$ of piperidine), $2.66(\mathrm{~m}, 4 \mathrm{H}$ of $\mathrm{C}-2$ and $\mathrm{C}-6$ of piperidine), $4.24(\mathrm{~d}, \mathrm{~J}=12 \mathrm{~Hz}$, $\left.2 \mathrm{H},-\mathrm{NH}-\mathrm{CH}_{2}-\mathrm{N}-\right)$, 6.96-7.65 (m, 10H, Ar-H), 7.86 (s, $1 \mathrm{H}, \overline{\mathrm{NH}}$, exchangable with $\left.\mathrm{D}_{2} \mathrm{O}\right) ;{ }^{13} \mathrm{C}-\mathrm{NMR}$ (DMSO) $\delta$ ppm: 26.4 (C-4 of piperidine), 27.5 (2C, C-3 \& C-5 of piperidine), 53.4 (2C, C-2 \& C6 of piperidine), $72.5\left(\mathrm{~N}-\mathrm{CH}_{2}-\mathrm{N}\right), 109.2,115.1$, $119.9,124.4,126.9,127.3,127.9$ (2C), 128.3 (2C), 128.4, 132.9, 133.3, 145.1, 152.0, 160.8, 160.9, 161.6, 164.0; Elemental Analysis $\left(\mathrm{C}_{25} \mathrm{H}_{23} \mathrm{ClN}_{4} \mathrm{O}_{2}\right)$, Found\% (Calculated\%): C, 67.15 (67.18); H, 5.15 (5.19); N, 12.50 (12.54).

\section{2-(Piperidinomethylamino)-4-(coumarin-3-yl)- 6-(2,6-dichlorophenyl) pyrimidine (2a)}

Yield: $45 \%$; m.p.: $170-172{ }^{\circ} \mathrm{C}$; $\mathrm{R}_{\mathrm{f}}$ : 0.71; IR (KBr) $\mathrm{cm}^{-1}$ : $3285(\mathrm{~N}-\mathrm{H}), 1708(\mathrm{C}=\mathrm{O}), 1610(\mathrm{C}=\mathrm{N}), 1541$ $(\mathrm{C}=\mathrm{C}), 1131$ (C-O-C); $\mathrm{H}-\mathrm{NMR}\left(\mathrm{CDCl}_{3}\right.$, DMSO$\left.\mathrm{d}_{6}\right) \delta$ ppm: $1.41(\mathrm{~m}, 2 \mathrm{H}$ of $\mathrm{C}-4$ of piperidine), 1.56 (m, $4 \mathrm{H}$ of $\mathrm{C}-3$ and $\mathrm{C}-5$ of piperidine), $2.67(\mathrm{~m}, 4 \mathrm{H}$ of $\mathrm{C}-2$ and $\mathrm{C}-6$ of piperidine), $4.25(\mathrm{~d}, \mathrm{~J}=12 \mathrm{~Hz}$, $\left.2 \mathrm{H},-\mathrm{NH}-\mathrm{CH}_{2}-\mathrm{N}-\right)$, 6.96-7.65 (m, 9H, Ar-H), 7.86 (s, $1 \mathrm{H}, \mathrm{NH}$, exchangable with $\mathrm{D}_{2} \mathrm{O}$ ); ${ }^{13} \mathrm{C}-\mathrm{NMR}$ (DMSO) $\delta$ ppm: 26.3 (C-4 of piperidine), 27.4 (2C, C-3 \& C-5 of piperidine), 53.4 (2C, C-2 \& C6 of piperidine), $72.4\left(\mathrm{~N}-\mathrm{CH}_{2}-\mathrm{N}\right), 109.2,115.1$, $119.9,124.4,126.2,126.9,127.3,128.4,129.5$, 130.9 (2C), 132.6 (2C), 145.1, 152.0, 160.8, 160.9, 161.6, 164.0; Elemental Analysis $\left(\mathrm{C}_{25} \mathrm{H}_{22} \mathrm{Cl}_{2} \mathrm{~N}_{4} \mathrm{O}_{2}\right)$, Found\% (Calculated \%): C, 62.35 (62.38); H, 4.61 (4.61); N, 11.62 (11.64); Mass (m/z): $480\left(\mathrm{M}^{+}\right), 481\left(\mathrm{M}^{+}+1\right), 482\left(\mathrm{M}^{+}+2\right)$.

\section{2-(Piperidinomethylamino)-4-(coumarin-3-yl)-} 6-(2,4-dichlorophenyl) pyrimidine (3a)

Yield: 60 \%; m.p.: $172-174{ }^{\circ} \mathrm{C}$; $\mathrm{R}_{\mathrm{f}}$ : 0.77; IR (KBr) $\mathrm{cm}^{-1}$ : $3286(\mathrm{~N}-\mathrm{H}), 1709$ (C=O), $1605(\mathrm{C}=\mathrm{N}), 1540$ $(\mathrm{C}=\mathrm{C}), 1132$ (C-O-C); $\mathrm{H}-\mathrm{NMR}\left(\mathrm{CDCl}_{3}\right.$, DMSO$\left.\mathrm{d}_{6}\right) \delta \mathrm{ppm}: 1.40(\mathrm{~m}, 2 \mathrm{H}$ of $\mathrm{C}-4$ of piperidine $), 1.57$ (m, $4 \mathrm{H}$ of $\mathrm{C}-3$ and $\mathrm{C}-5$ of piperidine), $2.66(\mathrm{~m}, 4 \mathrm{H}$ of C-2 and C-6 of piperidine), $4.24(\mathrm{~d}, \mathrm{~J}=12 \mathrm{~Hz}$, $\left.2 \mathrm{H},-\mathrm{NH}-\mathrm{CH}_{2}-\mathrm{N}-\right), 6.95-7.68(\mathrm{~m}, 9 \mathrm{H}, \mathrm{Ar}-\mathrm{H}), 7.86$ (s, $1 \mathrm{H}, \widehat{\mathrm{NH}}$, exchangable with $\mathrm{D}_{2} \mathrm{O}$ ); ${ }^{13} \mathrm{C}-\mathrm{NMR}$ (DMSO) $\delta$ ppm: 26.4 (C-4 of piperidine), 27.5 (2C, C-3 \& C-5 of piperidine), 53.5 (2C, C-2 \& C6 of piperidine), $72.5\left(\mathrm{~N}-\mathrm{CH}_{2}-\mathrm{N}\right), 109.2,115.1$, $119.9,124.4,126.4,126.9,127.0,127.3,128.4$, 129.3 (2C), 132.6, 134.7, 145.1, 152.0, 160.6, 160.8, 161.6, 164.0; Elemental Analysis $\left(\mathrm{C}_{25} \mathrm{H}_{22} \mathrm{Cl}_{2} \mathrm{~N}_{4} \mathrm{O}_{2}\right)$, Found\% (Calculated\%): C, 62.35 (62.38); H, 4.60 (4.61); N, 11.61 (11.64).

\section{2-(Piperidinomethylamino)-4-(coumarin-3-yl)- 6-(2-chlorophenyl) pyrimidine (4a)}

Yield: 50 \%; m.p.: $155-157{ }^{\circ} \mathrm{C}$; $\mathrm{R}_{\mathrm{f}}$ : 0.66; IR (KBr) $\mathrm{cm}^{-1}: 3284(\mathrm{~N}-\mathrm{H}), 1709$ (C=O), $1608(\mathrm{C}=\mathrm{N}), 1542$ $(\mathrm{C}=\mathrm{C}), 1130$ (C-O-C); $\mathrm{H}-\mathrm{NMR}\left(\mathrm{CDCl}_{3}\right.$, DMSO$\left.\mathrm{d}_{6}\right) \delta$ ppm: $1.40(\mathrm{~m}, 2 \mathrm{H}$ of $\mathrm{C}-4$ of piperidine $), 1.57$ ( $\mathrm{m}, 4 \mathrm{H}$ of $\mathrm{C}-3$ and $\mathrm{C}-5$ of piperidine), $2.66(\mathrm{~m}, 4 \mathrm{H}$ of $\mathrm{C}-2$ and $\mathrm{C}-6$ of piperidine), 4.24 (d, J = $12 \mathrm{~Hz}$, $\left.2 \mathrm{H},-\mathrm{NH}-\mathrm{CH}_{2}-\mathrm{N}-\right)$, 6.94-7.70 (m, 10H, Ar-H), 7.85 (s, $1 \mathrm{H}, \mathrm{NH}$, exchangable with $\mathrm{D}_{2} \mathrm{O}$ ); ${ }^{13} \mathrm{C}-\mathrm{NMR}$ (DMSO) $\delta$ ppm: 26.5 (C-4 of piperidine), 27.4 (2C, C-3 \& C-5 of piperidine), 53.4 (2C, C-2 \& C6 of piperidine), $72.4\left(\mathrm{~N}-\mathrm{CH}_{2}-\mathrm{N}\right), 109.2,115.1$, $119.9,124.4,126.4,126.9,127.0,127.3,128.4$, $129.1,129.3,129.9,132.6,134.7,145.1,152.0$, 160.8, 161.6, 164.0.; Elemental Analysis $\left(\mathrm{C}_{25} \mathrm{H}_{23} \mathrm{ClN}_{4} \mathrm{O}_{2}\right)$, Found\% (Calculated\%): C, 67.15 (67.18); H, 5.17 (5.19); N, 12.55 (12.54); Mass $(\mathrm{m} / \mathrm{z}): 446\left(\mathrm{M}^{+}\right), 447\left(\mathrm{M}^{+}+1\right)$.

2-(Piperidinomethylamino)-4-(7chlorocoumarin-3-yl)-6-(4-chlorophenyl) pyrimidine (5a)

Yield: $45 \%$; m.p.: $155-157^{\circ} \mathrm{C}$; $\mathrm{R}_{\mathrm{f}}$ : 0.77; IR (KBr) $\mathrm{cm}^{-1}$ : $3285(\mathrm{~N}-\mathrm{H}), 1709$ (C=O), $1605(\mathrm{C}=\mathrm{N}), 1545$ $(\mathrm{C}=\mathrm{C}), 1133$ (C-O-C); ${ }^{1} \mathrm{H}-\mathrm{NMR}\left(\mathrm{CDCl}_{3}\right.$, DMSO$\left.\mathrm{d}_{6}\right) \delta$ ppm: $1.41(\mathrm{~m}, 2 \mathrm{H}$ of $\mathrm{C}-4$ of piperidine $), 1.56$ (m, $4 \mathrm{H}$ of $\mathrm{C}-3$ and $\mathrm{C}-5$ of piperidine), $2.67(\mathrm{~m}, 4 \mathrm{H}$ of $\mathrm{C}-2$ and $\mathrm{C}-6$ of piperidine), $4.24(\mathrm{~d}, \mathrm{~J}=12 \mathrm{~Hz}$, $\left.2 \mathrm{H},-\mathrm{NH}-\mathrm{CH}_{2}-\mathrm{N}-\right)$, 6.95-7.68 (m, 9H, Ar-H), 7.82 (s, $1 \mathrm{H}, \mathrm{NH}$, exchangable with $\mathrm{D}_{2} \mathrm{O}$ ); ${ }^{13} \mathrm{C}-\mathrm{NMR}$ (DMSO) $\delta$ ppm: 26.4 (C-4 of piperidine), 27.5 (2C, C-3 \& C-5 of piperidine), 53.5 (2C, C-2 \& C6 of piperidine), $72.5\left(\mathrm{~N}-\mathrm{CH}_{2}-\mathrm{N}\right), 109.2,118.0$, 118.6, 124.5, 127.9 (2C), 128.3 (4C), 132.9, 133.3, 134.1, 145.1, 153.5, 160.8 (2C), 161.6, 164.0; Elemental Analysis $\left(\mathrm{C}_{25} \mathrm{H}_{22} \mathrm{Cl}_{2} \mathrm{~N}_{4} \mathrm{O}_{2}\right)$, Found\% (Calculated\%): C, 62.35 (62.38); H, 4.62 (4.61); N, 11.62 (11.64).

\section{2-(Piperidinomethylamino)-4-(7- bromocoumarin-3-yl)-6-(4-chlorophenyl) pyrimidine (6a)}

Yield: 55 \%; m.p.: 190-192 ${ }^{\circ} \mathrm{C}$; $\mathrm{R}_{\mathrm{f}}$ : 0.71; IR (KBr) $\mathrm{cm}^{-1}$ : $3285(\mathrm{~N}-\mathrm{H}), 1712(\mathrm{C}=\mathrm{O}), 1607(\mathrm{C}=\mathrm{N}), 1541$ 
$(\mathrm{C}=\mathrm{C}), 1131$ (C-O-C); ${ }^{1} \mathrm{H}-\mathrm{NMR}\left(\mathrm{CDCl}_{3}\right.$, DMSO$\left.\mathrm{d}_{6}\right) \delta \mathrm{ppm}: 1.40(\mathrm{~m}, 2 \mathrm{H}$ of C-4 of piperidine), 1.57 ( $\mathrm{m}, 4 \mathrm{H}$ of $\mathrm{C}-3$ and $\mathrm{C}-5$ of piperidine), $2.66(\mathrm{~m}, 4 \mathrm{H}$ of $\mathrm{C}-2$ and $\mathrm{C}-6$ of piperidine), $4.24(\mathrm{~d}, \mathrm{~J}=12 \mathrm{~Hz}$, $\left.2 \mathrm{H},-\mathrm{NH}-\mathrm{CH}_{2}-\mathrm{N}-\right)$, 6.93-7.65 (m, 9H, Ar-H), 7.82 (s, $1 \mathrm{H}, \mathrm{NH}$, exchangable with $\mathrm{D}_{2} \mathrm{O}$ ); ${ }^{13} \mathrm{C}-\mathrm{NMR}$ (DMSO) $\delta$ ppm: 26.4 (C-4 of piperidine), 27.5 (2C, C-3 \& C-5 of piperidine), 53.5 (2C, C-2 \& C6 of piperidine), $72.5\left(\mathrm{~N}-\mathrm{CH}_{2}-\mathrm{N}\right), 109.2,118.8$ (2C), 124.5, 127.3, 127.9 (3C), 128.3 (3C), 132.9, 133.3, 145.1, 153.1, 160.8, 160.9, 161.6, 164.0; Elemental Analysis $\left(\mathrm{C}_{25} \mathrm{H}_{22} \mathrm{BrClN}_{4} \mathrm{O}_{2}\right)$, Found \% (Calculated\%): C, $57.12(57.10) ; \mathrm{H}$, 4.20 (4.22); N, 10.64 (10.66); Mass (m/z): 524 $\left(\mathrm{M}^{+}\right), 525\left(\mathrm{M}^{+}+1\right), 526\left(\mathrm{M}^{+}+2\right)$.

\section{2-(Piperidinomethylamino)-4-(7- chlorocoumrin-3-yl)-6-(2,6-dichlorophenyl) pyrimidine (7a)}

Yield: 40 \%; m.p.: $158-160{ }^{\circ} \mathrm{C}$; $\mathrm{R}_{\mathrm{f}}$ : 0.74; IR (KBr) $\mathrm{cm}^{-1}: 3288(\mathrm{~N}-\mathrm{H}), 1709(\mathrm{C}=\mathrm{O}), 1605(\mathrm{C}=\mathrm{N}), 1545$ $(\mathrm{C}=\mathrm{C}), 1130(\mathrm{C}-\mathrm{O}-\mathrm{C})$; ${ }^{\mathrm{H}}-\mathrm{NMR}\left(\mathrm{CDCl}_{3}\right.$, DMSO$\left.\mathrm{d}_{6}\right) \delta \mathrm{ppm}: 1.41(\mathrm{~m}, 2 \mathrm{H}$ of C-4 of piperidine), 1.57 ( $\mathrm{m}, 4 \mathrm{H}$ of $\mathrm{C}-3$ and $\mathrm{C}-5$ of piperidine), $2.65(\mathrm{~m}, 4 \mathrm{H}$ of $\mathrm{C}-2$ and $\mathrm{C}-6$ of piperidine), $4.25(\mathrm{~d}, \mathrm{~J}=12 \mathrm{~Hz}$, $\left.2 \mathrm{H},-\mathrm{NH}-\mathrm{CH}_{2}-\mathrm{N}-\right)$, 6.93-7.65 (m, 8H, Ar- $\left.\mathrm{H}\right), 7.82$ (s, $1 \mathrm{H}, \mathrm{NH}$, exchangable with $\mathrm{D}_{2} \mathrm{O}$ ); ${ }^{13} \mathrm{C}-\mathrm{NMR}$ (DMSO) $\delta$ ppm: 26.4 (C-4 of piperidine), 27.5 (2C, C-3 \& C-5 of piperidine), 53.5 (2C, C-2 \& C6 of piperidine), $72.5\left(\mathrm{~N}-\mathrm{CH}_{2}-\mathrm{N}\right), 109.2,118.0$, 118.6, 124.5, 126.2, 128.3 (2C), 129.5, 130.9 (2C), $132.6(2 \mathrm{C}), 134.1,145.1,153.5,160.8$ (2C), 161.6, 164.0; Elemental Analysis $\left(\mathrm{C}_{25} \mathrm{H}_{21} \mathrm{Cl}_{3} \mathrm{~N}_{4} \mathrm{O}_{2}\right.$ ), Found \% (Calculated\%): $\mathrm{C}$, 58.20 (58.21); H, 4.08 (4.10); N, 10.88 (10.86).

\section{2-(Piperidinomethylamino)-4-(7- bromocoumarin-3-yl)-6-(2,6-dichlorophenyl) pyrimidine (8a)}

Yield: 50 \%; m.p.: $165-167{ }^{\circ} \mathrm{C}$; $\mathrm{R}_{\mathrm{f}}$ : 0.77; IR (KBr) $\mathrm{cm}^{-1}: 3288(\mathrm{~N}-\mathrm{H}), 1708(\mathrm{C}=\mathrm{O}), 1611(\mathrm{C}=\mathrm{N}), 1541$ $(\mathrm{C}=\mathrm{C}), 1130(\mathrm{C}-\mathrm{O}-\mathrm{C})$; ${ }^{1} \mathrm{H}-\mathrm{NMR}\left(\mathrm{CDCl}_{3}\right.$, DMSO$\left.\mathrm{d}_{6}\right) \delta \mathrm{ppm}: 1.40(\mathrm{~m}, 2 \mathrm{H}$ of C-4 of piperidine), 1.56 ( $\mathrm{m}, 4 \mathrm{H}$ of $\mathrm{C}-3$ and $\mathrm{C}-5$ of piperidine), $2.66(\mathrm{~m}, 4 \mathrm{H}$ of $\mathrm{C}-2$ and $\mathrm{C}-6$ of piperidine), $4.24(\mathrm{~d}, \mathrm{~J}=12 \mathrm{~Hz}$, $\left.2 \mathrm{H},-\mathrm{NH}-\mathrm{CH}_{2}-\mathrm{N}-\right), 6.98-7.61(\mathrm{~m}, 8 \mathrm{H}, \mathrm{Ar}-\mathrm{H}), 7.81$ (s, $1 \mathrm{H}, \mathrm{NH}$, exchangable with $\mathrm{D}_{2} \mathrm{O}$ ); ${ }^{13} \mathrm{C}-\mathrm{NMR}$ (DMSO) $\delta$ ppm: 26.5 (C-4 of piperidine), 27.4 (2C, C-3 \& C-5 of piperidine), 53.5 (2C, C-2 \& C6 of piperidine), $72.4\left(\mathrm{~N}-\mathrm{CH}_{2}-\mathrm{N}\right), 109.2,118.8$ (2C), 124.5, 126.2, 127.3, 128.0, 128.4, 129.5, 130.9 (2C), $132.6(2 \mathrm{C}), 145.1,153.1,160.8$ (2C), 161.6, 164.0; Elemental Analysis $\left(\mathrm{C}_{25} \mathrm{H}_{21} \mathrm{BrCl}_{2} \mathrm{~N}_{4} \mathrm{O}_{2}\right)$, Found \% (Calculated\%): C, 53.58 (53.59); H, 3.75 (3.78); N, 9.96 (10.0); Mass (m/z): $558\left(\mathrm{M}^{+}\right), 559\left(\mathrm{M}^{+}+1\right), 560\left(\mathrm{M}^{+}+2\right)$.
2-(Piperidinomethylamino)-4-(7chlorocoumarin-3-yl)-6-(2,4-dichlorophenyl) pyrimidine (9a)

Yield: 45 \%; m.p.: $148-150{ }^{\circ} \mathrm{C}$; $\mathrm{R}_{\mathrm{f}}$ : 0.67; IR (KBr) $\mathrm{cm}^{-1}: 3285(\mathrm{~N}-\mathrm{H}), 1708(\mathrm{C}=\mathrm{O}), 1607(\mathrm{C}=\mathrm{N}), 1543$ $(\mathrm{C}=\mathrm{C}), 1132(\mathrm{C}-\mathrm{O}-\mathrm{C})$; ${ }^{1} \mathrm{H}-\mathrm{NMR}\left(\mathrm{CDCl}_{3}\right.$, DMSO$\left.\mathrm{d}_{6}\right) \delta \mathrm{ppm}$ : $1.41(\mathrm{~m}, 2 \mathrm{H}$ of C-4 of piperidine), 1.55 ( $\mathrm{m}, 4 \mathrm{H}$ of $\mathrm{C}-3$ and $\mathrm{C}-5$ of piperidine), $2.65(\mathrm{~m}, 4 \mathrm{H}$ of $\mathrm{C}-2$ and $\mathrm{C}-6$ of piperidine), $4.25(\mathrm{~d}, \mathrm{~J}=12 \mathrm{~Hz}$, $\left.2 \mathrm{H},-\mathrm{NH}-\mathrm{CH}_{2}-\mathrm{N}-\right)$, 6.95-7.66 (m, 8H, Ar-H), 7.83 (s, $1 \mathrm{H}, \mathrm{NH}$, exchangable with $\mathrm{D}_{2} \mathrm{O}$ ); ${ }^{13} \mathrm{C}-\mathrm{NMR}$ (DMSO) $\delta$ ppm: 26.5 (C-4 of piperidine), 27.4 (2C, C-3 \& C-5 of piperidine), 53.5 (2C, C-2 \& C6 of piperidine), $72.4\left(\mathrm{~N}^{-} \mathrm{CH}_{2}-\mathrm{N}\right), 109.2,118.0$, 118.6, 124.5, 126.4, 127.0, 128.3 (2C), 129.3, $129.9,132.6,134.1,134.7,145.1,153.5,160.8$ (2C), 161.6, 164.0; Elemental Analysis $\left(\mathrm{C}_{25} \mathrm{H}_{21} \mathrm{Cl}_{3} \mathrm{~N}_{4} \mathrm{O}_{2}\right)$, Found \% (Calculated\%): C, 58.20 (58.21); H, 4.08 (4.10); N, 10.85 (10.86).

\section{2-(Piperidinomethylamino)-4-(7- bromocoumarin-3-yl)-6-(2,4-dichlorophenyl) pyrimidine (10a)}

Yield: 55 \%; m.p.: $166-168{ }^{\circ} \mathrm{C}$; $\mathrm{R}_{\mathrm{f}}$ : 0.77; IR $(\mathrm{KBr})$ $\mathrm{cm}^{-1}: 3284(\mathrm{~N}-\mathrm{H}), 1708(\mathrm{C}=\mathrm{O}), 1603(\mathrm{C}=\mathrm{N}), 1542$ $(\mathrm{C}=\mathrm{C}), 1131(\mathrm{C}-\mathrm{O}-\mathrm{C})$; ' $\mathrm{H}-\mathrm{NMR}\left(\mathrm{CDCl}_{3}\right.$, DMSO$\left.\mathrm{d}_{6}\right) \delta \mathrm{ppm}: 1.41(\mathrm{~m}, 2 \mathrm{H}$ of C-4 of piperidine), 1.56 ( $\mathrm{m}, 4 \mathrm{H}$ of $\mathrm{C}-3$ and $\mathrm{C}-5$ of piperidine), $2.66(\mathrm{~m}, 4 \mathrm{H}$ of $\mathrm{C}-2$ and $\mathrm{C}-6$ of piperidine), $4.23(\mathrm{~d}, \mathrm{~J}=12 \mathrm{~Hz}$, $\left.2 \mathrm{H},-\mathrm{NH}-\mathrm{CH}_{2}-\mathrm{N}-\right), 6.92-7.64(\mathrm{~m}, 8 \mathrm{H}, \mathrm{Ar}-\mathrm{H}), 7.83$ (s, $1 \mathrm{H}, \mathrm{NH}$, exchangable with $\mathrm{D}_{2} \mathrm{O}$ ); ${ }^{13} \mathrm{C}-\mathrm{NMR}$ (DMSO) $\delta$ ppm: 26.4 (C-4 of piperidine), 27.4 (2C, C-3 \& C-5 of piperidine), 53.4 (2C, C-2 \& C6 of piperidine $), 72.5\left(\mathrm{~N}^{-} \mathrm{CH}_{2}-\mathrm{N}\right), 109.2,118.8$ (2C), 124.5, 126.4, 127.0, 127.3, 128.0, 128.4, 129.3, 129.9, 132.6, 134.7, 145.1, 153.1, 160.8 (2C), 161.6, 164.0; Elemental Analysis $\left(\mathrm{C}_{25} \mathrm{H}_{21} \mathrm{BrCl}_{2} \mathrm{~N}_{4} \mathrm{O}_{2}\right)$, Found \% (Calculated\%): C, 53.58 (53.59); H, 3.77 (3.78); N, 9.98 (10.0).

\section{2-(Piperidinomethylamino)-4-(7- chlorocoumarin-3-yl)-6-(2-chlorophenyl) pyrimidine (11a)}

Yield: 60 \%; m.p.: $158-160{ }^{\circ} \mathrm{C}$; $\mathrm{R}_{\mathrm{f}}$ : 0.66; IR (KBr) $\mathrm{cm}^{-1}: 3285(\mathrm{~N}-\mathrm{H}), 1710(\mathrm{C}=\mathrm{O}), 1603(\mathrm{C}=\mathrm{N}), 1541$ $(\mathrm{C}=\mathrm{C}), 1133(\mathrm{C}-\mathrm{O}-\mathrm{C})$; 'H-NMR $\left(\mathrm{CDCl}_{3}\right.$, DMSO$\left.\mathrm{d}_{6}\right) \delta$ ppm: 1.41 (m, $2 \mathrm{H}$ of $\mathrm{C}-4$ of piperidine), 1.56 ( $\mathrm{m}, 4 \mathrm{H}$ of $\mathrm{C}-3$ and $\mathrm{C}-5$ of piperidine), $2.65(\mathrm{~m}, 4 \mathrm{H}$ of $\mathrm{C}-2$ and $\mathrm{C}-6$ of piperidine), $4.24(\mathrm{~d}, \mathrm{~J}=12 \mathrm{~Hz}$, $\left.2 \mathrm{H},-\mathrm{NH}-\mathrm{CH}_{2}-\mathrm{N}-\right)$, 6.95-7.67 (m, 9H, Ar-H), 7.84 (s, $1 \mathrm{H}, \mathrm{NH}$, exchangable with $\mathrm{D}_{2} \mathrm{O}$ ); ${ }^{13} \mathrm{C}-\mathrm{NMR}$ (DMSO) $\delta$ ppm: 26.5 (C-4 of piperidine), 27.4 (2C, C-3 \& C-5 of piperidine), 53.5 (2C, C-2 \& C6 of piperidine), $72.5\left(\mathrm{~N}-\mathrm{CH}_{2}-\mathrm{N}\right), 109.2,118.0$, 118.6, 124.5, 127.9, 128.3 (2C), 128.9, 129.1, 129.6, 131.2, 131.5, 134.1, 145.1, 153.5, 160.8 (2C), 161.6, 164.0; Elemental Analysis 
$\left(\mathrm{C}_{25} \mathrm{H}_{22} \mathrm{Cl}_{2} \mathrm{~N}_{4} \mathrm{O}_{2}\right)$, Found \% (Calculated \%): C, 62.35 (62.38); H, 4.56 (4.61); N, 11.60 (11.64); Mass (m/z): $480\left(\mathrm{M}^{+}\right), 481\left(\mathrm{M}^{+}+1\right), 482\left(\mathrm{M}^{+}+2\right)$.

\section{2-(Piperidinomethylamino)-4-(7- bromocoumarin-3-yl)-6-(2-chlorophenyl) pyrimidine (12a)}

Yield: 45 \%; m.p.: 167-168 ${ }^{\circ} \mathrm{C}$; $\mathrm{R}_{\mathrm{f}}$ : 0.66; IR (KBr) $\mathrm{cm}^{-1}$ : $3283(\mathrm{~N}-\mathrm{H}), 1709$ (C=O), $1608(\mathrm{C}=\mathrm{N}), 1545$ $(\mathrm{C}=\mathrm{C}), 1131$ (C-O-C); $\mathrm{H}-\mathrm{NMR}\left(\mathrm{CDCl}_{3}, \mathrm{DMSO}-\right.$ $\left.\mathrm{d}_{6}\right) \delta \mathrm{ppm}: 1.40(\mathrm{~m}, 2 \mathrm{H}$ of $\mathrm{C}-4$ of piperidine $), 1.56$ ( $\mathrm{m}, 4 \mathrm{H}$ of $\mathrm{C}-3$ and $\mathrm{C}-5$ of piperidine), $2.66(\mathrm{~m}, 4 \mathrm{H}$ of $\mathrm{C}-2$ and $\mathrm{C}-6$ of piperidine), $4.24(\mathrm{~d}, \mathrm{~J}=12 \mathrm{~Hz}$, $\left.2 \mathrm{H},-\mathrm{NH}-\mathrm{CH}_{2}-\mathrm{N}-\right)$, 6.94-7.66 (m, 9H, Ar-H), 7.83 (s, $1 \mathrm{H}, \mathrm{NH}$, exchangable with $\mathrm{D}_{2} \mathrm{O}$ ); ${ }^{13} \mathrm{C}-\mathrm{NMR}$ (DMSO) $\delta$ ppm: 26.4 (C-4 of piperidine), 27.4 (2C, C-3 \& C-5 of piperidine), 53.4 (2C, C-2 \& C6 of piperidine), $72.5\left(\mathrm{~N}-\mathrm{CH}_{2}-\mathrm{N}\right), 109.2,118.8$ (2C), 124.5, 127.3, 127.9, 128.0, 128.4, 128.9, $129.1,129.6,131.2,131.5,145.1,153.1,160.8$ (2C), 161.6, 164.0; Elemental Analysis $\left(\mathrm{C}_{25} \mathrm{H}_{22} \mathrm{BrClN}_{4} \mathrm{O}_{2}\right)$, Found \% (Calculated \%): C, 57.08 (57.10); H, 4.20 (4.22); N, 10.60 (10.66); Mass (m/z): $524\left(\mathrm{M}^{+}\right), 525\left(\mathrm{M}^{+}+1\right), 526\left(\mathrm{M}^{+}+2\right)$.

\section{2-(Piperidinomethylamino)-4-(7- chlorocoumarin-3-yl)-6-(2,5-dichlorophenyl) pyrimidine (13a)}

Yield: 65 \%; m.p.: 167-169 ${ }^{\circ} \mathrm{C}$; $\mathrm{R}_{\mathrm{f}}$ : 0.77; IR (KBr) $\mathrm{cm}^{-1}: 3286(\mathrm{~N}-\mathrm{H}), 1709$ (C=O), $1607(\mathrm{C}=\mathrm{N}), 1540$ $(\mathrm{C}=\mathrm{C}), 1131$ (C-O-C); $\mathrm{H}-\mathrm{NMR}\left(\mathrm{CDCl}_{3}\right.$, DMSO$\left.\mathrm{d}_{6}\right) \delta \mathrm{ppm}: 1.41(\mathrm{~m}, 2 \mathrm{H}$ of $\mathrm{C}-4$ of piperidine $), 1.56$ ( $\mathrm{m}, 4 \mathrm{H}$ of $\mathrm{C}-3$ and $\mathrm{C}-5$ of piperidine), $2.65(\mathrm{~m}, 4 \mathrm{H}$ of C-2 and C-6 of piperidine), 4.25 (d, $\mathrm{J}=12 \mathrm{~Hz}$, $\left.2 \mathrm{H},-\mathrm{NH}-\mathrm{CH}_{2}-\mathrm{N}-\right)$, 6.94-7.62 (m, 8H, Ar-H), 7.82 (s, $1 \mathrm{H}, \hat{\mathrm{NH}}$, exchangable with $\mathrm{D}_{2} \mathrm{O}$ ); ${ }^{13} \mathrm{C}-\mathrm{NMR}$ (DMSO) $\delta$ ppm: 26.5 (C-4 of piperidine), 27.4 (2C, C-3 \& C-5 of piperidine), 53.5 (2C, C-2 \& C6 of piperidine), $72.3\left(\mathrm{~N}-\mathrm{CH}_{2}-\mathrm{N}\right), 109.2,118.0$, $118.6,124.5,127.8,128.3$ (2C), 129.2 (2C), $129.7,130.3,131.9,134.1,145.1,153.5,160.8$ (2C), 161.6, 164.0; Elemental Analysis $\left(\mathrm{C}_{25} \mathrm{H}_{21} \mathrm{Cl}_{3} \mathrm{~N}_{4} \mathrm{O}_{2}\right)$, Found \% (Calculated\%): C, 58.18 (58.21); H, 4.07 (4.10); N, 10.83 (10.86).

\section{2-(Piperidinomethylamino)-4-(7- bromocoumarin-3-yl)-6-(2,5-dichlorophenyl) pyrimidine (14a)}

Yield: 45 \%; m.p.: $176-178{ }^{\circ} \mathrm{C}$; $\mathrm{R}_{\mathrm{f}}$ : 0.77; IR (KBr) $\mathrm{cm}^{-1}$ : $3286(\mathrm{~N}-\mathrm{H}), 1710(\mathrm{C}=\mathrm{O}), 1610(\mathrm{C}=\mathrm{N}), 1545$ $(\mathrm{C}=\mathrm{C}), 1133$ (C-O-C); $\mathrm{H}-\mathrm{NMR}\left(\mathrm{CDCl}_{3}\right.$, DMSO$\left.\mathrm{d}_{6}\right) \delta$ ppm: $1.41(\mathrm{~m}, 2 \mathrm{H}$ of $\mathrm{C}-4$ of piperidine $), 1.55$ ( $\mathrm{m}, 4 \mathrm{H}$ of $\mathrm{C}-3$ and $\mathrm{C}-5$ of piperidine $), 2.66(\mathrm{~m}, 4 \mathrm{H}$ of $\mathrm{C}-2$ and $\mathrm{C}-6$ of piperidine), $4.25(\mathrm{~d}, \mathrm{~J}=12 \mathrm{~Hz}$, $\left.2 \mathrm{H},-\mathrm{NH}-\mathrm{CH}_{2}-\mathrm{N}-\right)$, 6.93-7.66 (m, 8H, Ar-H), 7.81 (s, $1 \mathrm{H}, \mathrm{NH}$, exchangable with $\left.\mathrm{D}_{2} \mathrm{O}\right) ;{ }^{13} \mathrm{C}-\mathrm{NMR}$ (DMSO) $\delta$ ppm: 26.4 (C-4 of piperidine), 27.4
(2C, C-3 \& C-5 of piperidine), 53.4 (2C, C-2 \& C6 of piperidine $), 72.5\left(\mathrm{~N}-\mathrm{CH}_{2}-\mathrm{N}\right), 109.2,118.8$ (2C), 124.5, 127.3, 127.8, 128.0, 128.4, 129.2 (2C), 129.7, 130.3, 131.9, 145.1, 153.1, 160.8 (2C), 161.6, 164.0; Elemental Analysis $\left(\mathrm{C}_{25} \mathrm{H}_{21} \mathrm{BrCl}_{2} \mathrm{~N}_{4} \mathrm{O}_{2}\right)$, Found\% (Calculated\%): C, 53.55 (53.59); H, 3.75 (3.78); N, 9.98 (10.0).

\section{2-(Piperidinomethylamino)-4-(7- chlorocoumarin-3-yl)-6-(3,5-dichlorophenyl) pyrimidine (15a)}

Yield: 55 \%; m.p.: 177-179 ${ }^{\circ} \mathrm{C}$; $\mathrm{R}_{\mathrm{f}}$ : 0.74; IR (KBr) $\mathrm{cm}^{-1}: 3283(\mathrm{~N}-\mathrm{H}), 1709$ (C=O), $1605(\mathrm{C}=\mathrm{N}), 1543$ $(\mathrm{C}=\mathrm{C}), 1131$ (C-O-C); $\mathrm{H}-\mathrm{NMR}\left(\mathrm{CDCl}_{3}\right.$, DMSO$\left.\mathrm{d}_{6}\right) \delta \mathrm{ppm}: 1.40(\mathrm{~m}, 2 \mathrm{H}$ of $\mathrm{C}-4$ of piperidine $), 1.56$ ( $\mathrm{m}, 4 \mathrm{H}$ of $\mathrm{C}-3$ and $\mathrm{C}-5$ of piperidine), $2.66(\mathrm{~m}, 4 \mathrm{H}$ of $\mathrm{C}-2$ and $\mathrm{C}-6$ of piperidine), $4.25(\mathrm{~d}, \mathrm{~J}=12 \mathrm{~Hz}$, $\left.2 \mathrm{H},-\mathrm{NH}-\mathrm{CH}_{2}-\mathrm{N}-\right), 6.92-7.63$ (m, 8H, Ar-H), 7.80 (s, $1 \mathrm{H}, \mathrm{NH}$, exchangable with $\left.\mathrm{D}_{2} \mathrm{O}\right) ;{ }^{13} \mathrm{C}-\mathrm{NMR}$ (DMSO) $\delta$ ppm: 26.5 (C-4 of piperidine), 27.4 (2C, C-3 \& C-5 of piperidine), 53.5 (2C, C-2 \& C6 of piperidine), $72.5\left(\mathrm{~N}-\mathrm{CH}_{2}-\mathrm{N}\right), 109.2,118.0$, 118.6, 124.5, 126.7 (2C), 128.2 (2C), 128.4, 133.9 (2C), 134.1, 134.8, 145.1, 153.5, 160.8 (2C), 161.9, 164.0; Elemental Analysis $\left(\mathrm{C}_{25} \mathrm{H}_{21} \mathrm{Cl}_{3} \mathrm{~N}_{4} \mathrm{O}_{2}\right)$, Found\% (Calculated\%): C, 58.18 (58.21); H, 4.07 (4.10); N, 10.83 (10.86).

\section{2-(Piperidinomethylamino)-4-(7- bromocoumarin-3-yl)-6-(3,5-dichlorophenyl) pyrimidine (16a)}

Yield: 45 \%; m.p.: 158-160 ${ }^{\circ} \mathrm{C}$; $\mathrm{R}_{\mathrm{f}}$ : 0.66; IR (KBr) $\mathrm{cm}^{-1}$ : $3284(\mathrm{~N}-\mathrm{H}), 1709$ (C=O), $1611(\mathrm{C}=\mathrm{N}), 1542$ $(\mathrm{C}=\mathrm{C}), 1133$ (C-O-C); $\mathrm{H}-\mathrm{NMR}\left(\mathrm{CDCl}_{3}\right.$, DMSO$\left.\mathrm{d}_{6}\right) \delta$ ppm: $1.41(\mathrm{~m}, 2 \mathrm{H}$ of $\mathrm{C}-4$ of piperidine $), 1.56$ (m, 4H of C-3 and C-5 of piperidine), $2.66(\mathrm{~m}, 4 \mathrm{H}$ of $\mathrm{C}-2$ and $\mathrm{C}-6$ of piperidine), $4.25(\mathrm{~d}, \mathrm{~J}=12 \mathrm{~Hz}$, $\left.2 \mathrm{H},-\mathrm{NH}-\mathrm{CH}_{2}-\mathrm{N}-\right)$, 6.91-7.63 (m, 8H, Ar-H), 7.81 (s, $1 \mathrm{H}, \mathrm{NH}$, exchangable with $\mathrm{D}_{2} \mathrm{O}$ ); ${ }^{13} \mathrm{C}-\mathrm{NMR}$ (DMSO) $\delta$ ppm: 26.5 (C-4 of piperidine), 27.5 (2C, C-3 \& C-5 of piperidine), 53.5 (2C, C-2 \& C6 of piperidine), $72.5\left(\mathrm{~N}-\mathrm{CH}_{2}-\mathrm{N}\right), 109.2,118.8$ (2C), 124.5, 126.7 (2C), 127.3, 128.0, 128.2 (2C), 133.9 (2C), 134.8, 145.1, 153.1, 160.8 (2C), 161.6, 165.0; Elemental Analysis $\left(\mathrm{C}_{25} \mathrm{H}_{21} \mathrm{BrCl}_{2} \mathrm{~N}_{4} \mathrm{O}_{2}\right)$, Found \% (Calculated \%): C, 53.55 (53.59); H, 3.75 (3.78); N, 9.97 (10.0).

\section{2-(Piperidinomethylamino)-4-(7- chlorocoumarin-3-yl)-6-(3,4-dichlorophenyl) pyrimidine (17a)}

Yield: 55 \%; m.p.: $166-168{ }^{\circ} \mathrm{C}$; $\mathrm{R}_{\mathrm{f}}$ : 0.68; IR ( $\left.\mathrm{KBr}\right)$ $\mathrm{cm}^{-1}$ : $3284(\mathrm{~N}-\mathrm{H}), 1708(\mathrm{C}=\mathrm{O}), 1604(\mathrm{C}=\mathrm{N}), 1540$ $(\mathrm{C}=\mathrm{C}), 1133$ (C-O-C); $\mathrm{H}-\mathrm{NMR}\left(\mathrm{CDCl}_{3}\right.$, DMSO$\left.\mathrm{d}_{6}\right) \delta \mathrm{ppm}: 1.40(\mathrm{~m}, 2 \mathrm{H}$ of $\mathrm{C}-4$ of piperidine $), 1.56$ ( $\mathrm{m}, 4 \mathrm{H}$ of $\mathrm{C}-3$ and $\mathrm{C}-5$ of piperidine), $2.65(\mathrm{~m}, 4 \mathrm{H}$ of C-2 and C-6 of piperidine), 4.25 (d, J = 12Hz, 
$\left.2 \mathrm{H},-\mathrm{NH}-\mathrm{CH}_{2}-\mathrm{N}-\right), 6.92-7.65(\mathrm{~m}, 8 \mathrm{H}, \mathrm{Ar}-\mathrm{H}), 7.82$ (s, $1 \mathrm{H}, \hat{\mathrm{NH}}$, exchangable with $\mathrm{D}_{2} \mathrm{O}$ ); ${ }^{13} \mathrm{C}-\mathrm{NMR}$ (DMSO) $\delta$ ppm: 26.5 (C-4 of piperidine), 27.5 (2C, C-3 \& C-5 of piperidine), 53.5 (2C, C-2 \& C6 of piperidine $), 72.5\left(\mathrm{~N}-\mathrm{CH}_{2}-\mathrm{N}\right), 109.2,118.0$, $118.6,124.5,126.0,127.8,128.3$ (2C), 129.7, $131.5,131.7,132.4,134.1,145.1,153.5,160.8$ (2C), 161.6, 164.0; Elemental Analysis $\left(\mathrm{C}_{25} \mathrm{H}_{21} \mathrm{Cl}_{3} \mathrm{~N}_{4} \mathrm{O}_{2}\right)$, Found \% (Calculated \%): C, 58.17 (58.21); H, 4.07 (4.10); N, 10.83 (10.86).

\section{2-(Piperidinomethylamino)-4-(7- bromocoumarin-3-yl)-6-(3,4-dichlorophenyl) pyrimidine (18a)}

Yield: 60 \%; m.p.: $177-179{ }^{\circ} \mathrm{C}$; $\mathrm{R}_{\mathrm{f}}$ : 0.66; IR (KBr) $\mathrm{cm}^{-1}: 3285(\mathrm{~N}-\mathrm{H}), 1710(\mathrm{C}=\mathrm{O}), 1606(\mathrm{C}=\mathrm{N}), 1545$ $(\mathrm{C}=\mathrm{C}), 1133$ (C-O-C); $\mathrm{H}-\mathrm{NMR}\left(\mathrm{CDCl}_{3}\right.$, DMSO$\left.\mathrm{d}_{6}\right) \delta \mathrm{ppm}: 1.40(\mathrm{~m}, 2 \mathrm{H}$ of $\mathrm{C}-4$ of piperidine $), 1.55$ ( $\mathrm{m}, 4 \mathrm{H}$ of $\mathrm{C}-3$ and $\mathrm{C}-5$ of piperidine), $2.66(\mathrm{~m}, 4 \mathrm{H}$ of $\mathrm{C}-2$ and $\mathrm{C}-6$ of piperidine), $4.25(\mathrm{~d}, \mathrm{~J}=12 \mathrm{~Hz}$, $\left.2 \mathrm{H},-\mathrm{NH}-\mathrm{CH}_{2}-\mathrm{N}-\right)$, 6.93-7.66 (m, 8H, Ar-H), 7.81 (s, $1 \mathrm{H}, \mathrm{NH}$, exchangable with $\left.\mathrm{D}_{2} \mathrm{O}\right) ;{ }^{13} \mathrm{C}-\mathrm{NMR}$ (DMSO) $\delta$ ppm: 26.5 (C-4 of piperidine), 27.5 (2C, C-3 \& C-5 of piperidine), 53.5 (2C, C-2 \& C6 of piperidine $), 72.5\left(\mathrm{~N}-\mathrm{CH}_{2}-\mathrm{N}\right), 109.2,118.8$ (2C), 124.5, 127.3, 127.8, 128.0, 128.4, 129.7, $131.5,131.7,132.4,134.1,145.1,153.1,160.8$ (2C), 161.6, 164.0; Elemental Analysis $\left(\mathrm{C}_{25} \mathrm{H}_{21} \mathrm{BrCl}_{2} \mathrm{~N}_{4} \mathrm{O}_{2}\right)$, Found \% (Calculated \%): C, 53.55 (53.59); H, 3.75 (3.78); N, 9.97 (10.0); Mass (m/z): $558\left(\mathrm{M}^{+}\right), 559\left(\mathrm{M}^{+}+1\right), 560\left(\mathrm{M}^{+}+2\right)$.

\section{Antimicrobial activity}

The antimicrobial activity data of the title compounds (1a-18a) at different concentrations against Gram positive bacteria, Gram negative bacteria and fungi is provided in Table 1, Table 2 and Table 3, respectively. The zone of inhibition produced by the MIC of the standard drugs, ofloxacin and ketoconazole, has been considered as $100 \%$ for comparing the antibacterial activity and antifungal activity data of the title compounds (1a-18a), respectively.

The antibacterial activity of ofloxacin against Gram positive bacteria revealed that it has a MIC value of $25 \mu \mathrm{g} / \mathrm{mL}$ against $S$. aureus, $E$. faecalis and $S$. epidermidis; and it has a MIC value of $12.5 \mu \mathrm{g} / \mathrm{mL}$ against $B$. subtilis and $B$. cereus. The antibacterial activity of the title compounds (1a18a) with respect to ofloxacin revealed that the compound 6a (MIC = $50 \mu \mathrm{g} / \mathrm{mL} ; p<0.0001$ ) displayed highest activity of about $106.95 \%$, $94.39 \%, 100.50 \%, 100.26 \%$, and $94.08 \%$ against $S$. aureus, $E$. faecalis, $S$. epidermidis, $B$. subtilis, and $B$. cereus, respectively. The compound 13a (MIC = $25 \mu \mathrm{g} / \mathrm{mL} ; p<0.0001$ ) and 14a $(\mathrm{MIC}=50 \mathrm{\mu g} / \mathrm{mL} ; p<0.05)$ also displayed $101.47 \%$ and $100.64 \%$ activity against $S$. aureus, respectively. Other compounds did not produce noticeable antibacterial activity against Gram positive bacteria even at higher concentrations with respect to ofloxacin.

The antibacterial activity of ofloxacin against Gram negative bacteria revealed that it has a MIC value of $12.5 \mu \mathrm{g} / \mathrm{mL}$ against $E$. coli; $P$. aeruginosa $K$. pneumonia and $P$. vulgaris; and it has a MIC value of $25 \mu \mathrm{g} / \mathrm{mL}$ against $B$. bronchiseptica. The antibacterial activity of the title compounds (1a-18a) with respect to ofloxacin revealed that the compound $6 \mathbf{a}$ (MIC = $25 \mu \mathrm{g} / \mathrm{mL} ; p<0.0001)$ displayed very good activity of about $102.37 \%, 102.20 \%, 101.52 \%$, $99.44 \%$, and $107.71 \%$ against $E$. coli; $P$. aeruginosa K. pneumonia, B. bronchiseptica, and $P$. vulgaris, respectively. The compound 10a (MIC $=50 \mu \mathrm{g} / \mathrm{mL} ; p<0.0001$ ) and compound 7a $(\mathrm{MIC}=25 \mu \mathrm{g} / \mathrm{mL} ; p<0.05)$ also exhibited highest activity of $102.51 \%$ and $105.38 \%$, respectively, against $P$. aeruginosa and $K$. pneumonia with $p<0.05$ or less. Other compounds did not produce noticeable antibacterial activity against Gram negative bacteria even at higher concentrations with respect to ofloxacin.

The antifungal activity of ketoconazole against fungi revealed that it has a MIC value of 12.5 $\mu \mathrm{g} / \mathrm{mL}$ against $C$. albicans, $A$. niger and $M$. purpureous; and it has a MIC value of $25 \mu \mathrm{g} / \mathrm{mL}$ against $A$. flavus and $P$. citrinum. The antifungal activity of the title compounds (1a-18a) with respect to ketoconazole revealed that the compound 6a (MIC = $25 \mu \mathrm{g} / \mathrm{mL} ; p<0.0001)$ produced very good activity of about $102.70 \%$, $105.70 \%, 114.09 \%, 104.02 \%$, and $134.89 \%$ against $C$. albicans, $A$. niger, $A$. flavus, $M$. purpureous, and $P$. citrinum, respectively. The compound 17a (MIC = $25 \mu \mathrm{g} / \mathrm{mL} ; p<0.0001$ ) also showed very good activity of about 108.43 $\%, 126.75 \%, 109.45 \%, 118.31 \%$, and 130.32 $\%$ against $C$. albicans, $A$. niger, $A$. flavus, $M$. purpureous, and $P$. citrinum, respectively. The compounds 1a (MIC = $25 \mu \mathrm{g} / \mathrm{mL} ; p<0.05)$, 4a $(\mathrm{MIC}=25 \mu \mathrm{g} / \mathrm{mL} ; p<0.0001), 9 \mathrm{a}(\mathrm{MIC}=25$ $\mu \mathrm{g} / \mathrm{mL} ; p<0.0001), 11 \mathrm{a}(\mathrm{MIC}=25 \mu \mathrm{g} / \mathrm{mL} ; p<$ $0.0001), 12 \mathrm{a}(\mathrm{MIC}=25 \mu \mathrm{g} / \mathrm{mL} ; p<0.0001), 14 \mathrm{a}$ $(\mathrm{MIC}=25 \mu \mathrm{g} / \mathrm{mL} ; p<0.05), 16 \mathrm{a}(\mathrm{MIC}=25$ $\mu \mathrm{g} / \mathrm{mL} ; p<0.0001)$, and 18a (MIC = $25 \mu \mathrm{g} / \mathrm{mL} ; p$ $<0.0001)$ produced better antifungal activity against $A$. niger than standard drug ketoconazole.

Compounds $2 \mathrm{a}(\mathrm{MIC}=25 \mu \mathrm{g} / \mathrm{mL} ; p<0.05)$, and 15a $(\mathrm{MIC}=25 \mu \mathrm{g} / \mathrm{mL} ; p<0.0001)$ produced better antifungal activity against $A$. flavus than 
standard drug ketoconazole. Compounds 3a $(\mathrm{MIC}=25 \mu \mathrm{g} / \mathrm{mL} ; p<0.0001), 11 \mathrm{a}(\mathrm{MIC}=25$ $\mu \mathrm{g} / \mathrm{mL} ; p<0.0001), 14 \mathrm{a}(\mathrm{MIC}=25 \mu \mathrm{g} / \mathrm{mL} ; p<$ $0.0001)$, and 16a (MIC $=25 \mu \mathrm{g} / \mathrm{mL} ; p<0.0001)$ displayed better antifungal activity against $M$. purpureous than standard drug ketoconazole. Compounds 7a (MIC $=25 \mu \mathrm{g} / \mathrm{mL} ; p<0.0001$ ), 9a $(\mathrm{MIC}=25 \mu \mathrm{g} / \mathrm{mL} ; p<0.0001), 10 a(\mathrm{MIC}=25$ $\mu \mathrm{g} / \mathrm{mL} ; p<0.0001), 11 \mathrm{a}(\mathrm{MIC}=25 \mu \mathrm{g} / \mathrm{mL} ; p<$ $0.001), 14 \mathrm{a}(\mathrm{MIC}=25 \mu \mathrm{g} / \mathrm{mL} ; p<0.0001), 15 \mathrm{a}$ $(\mathrm{MIC}=25 \mu \mathrm{g} / \mathrm{mL} ; p<0.05)$, and 18a (MIC $=25$ $\mu \mathrm{g} / \mathrm{mL} ; p<0.0001$ ) also showed better antifungal activity against $P$. citrinum than standard drug ketoconazole. Other compounds did not produce noticeable antifungal activity even at higher concentrations with respect to standard drug ketoconazole.

\section{DISCUSSION}

A total of eighteen new compounds (1a-18a) were successfully synthesized, and their structures were confirmed on the basis of their IR, ${ }^{1} \mathrm{H}$-NMR, ${ }^{13} \mathrm{C}-\mathrm{NMR}$, Mass and elemental analysis data. The characteristic peaks in $1 \mathrm{H}$ NMR spectra that confirmed the formation of the compounds (1a-18a) from the compounds (1-18) [24] and their corresponding morpholine derivatives [25] were the appearance of the signals at $\delta(\mathrm{ppm})$ values from 1.40 to 1.41 for the two protons of C-4 of piperidine ring; disappearance of the signals at $\delta(\mathrm{ppm})$ values from 2.63 to 2.72 and from 3.50 to 3.58 [25]; and the disappearance of the signals at $\delta(\mathrm{ppm})$ values from 5.33 to 5.38 [24].

Table 1: Antibacterial activity data of the title compounds (1a-18a) against Gram positive bacteria

\begin{tabular}{|c|c|c|c|c|c|}
\hline \multirow{2}{*}{ Compound } & \multicolumn{5}{|c|}{ Zone of inhibition $(\mathrm{mm} \pm \mathrm{SD})$ with corresponding MIC $(\mu \mathrm{g} / \mathrm{mL})$ in brackets } \\
\hline & S. aureus & E. faecalis & S. epidermidis & B. subtilis & B. cereus \\
\hline $1 a$ & $23.78 \pm 0.45^{a}(50)$ & $25.54 \pm 0.41^{a}(50)$ & $27.59 \pm 0.38^{a}(50)$ & $21.97 \pm 0.36^{a}(50)$ & $23.26 \pm 0.44^{a}(50)$ \\
\hline $2 a$ & $16.28 \pm 0.46^{a}(25)$ & $\begin{array}{c}13.47 \pm 0.37^{a} \\
(100)\end{array}$ & $20.51 \pm 0.38^{a}(50)$ & $21.45 \pm 0.34^{a}(50)$ & $\begin{array}{c}10.15 \pm 0.43^{a} \\
(100)\end{array}$ \\
\hline $3 a$ & $\begin{array}{c}10.69 \pm 0.47^{a} \\
(100)\end{array}$ & $18.31 \pm 0.42^{a}(75)$ & $\begin{array}{c}15.43 \pm 0.34^{a} \\
(100)\end{array}$ & $\begin{array}{c}13.28 \pm 0.40^{a} \\
(100)\end{array}$ & $22.02 \pm 0.36^{a}(50)$ \\
\hline $4 a$ & $22.68 \pm 0.36^{a}(50)$ & $\begin{array}{c}12.98 \pm 0.41^{a} \\
(100)\end{array}$ & $\begin{array}{c}10.21 \pm 0.44^{a} \\
(100)\end{array}$ & $18.72 \pm 0.46^{a}(75)$ & $25.93 \pm 0.45^{a}(25)$ \\
\hline $5 a$ & $24.06 \pm 0.47^{d}(50)$ & $23.24 \pm 0.40^{a}(50)$ & $21.23 \pm 0.42^{a}(50)$ & $27.69 \pm 0.49^{a}(50)$ & $25.08 \pm 0.37^{a}(50)$ \\
\hline $6 a$ & $28.29 \pm 0.40^{C}(50)$ & $26.42 \pm 0.43^{a}(50)$ & $29.70 \pm 0.29^{c}(50)$ & $30.74 \pm 0.32^{a}(50)$ & $29.07 \pm 0.40^{a}(25)$ \\
\hline $7 a$ & $22.22 \pm 0.38^{a}(50)$ & $20.71 \pm 0.45^{a}(75)$ & $26.42 \pm 0.28^{a}(50)$ & $\begin{array}{c}18.45 \pm 0.44^{a} \\
(100)\end{array}$ & $21.06 \pm 0.45^{a}(75)$ \\
\hline $8 a$ & $\begin{array}{c}13.51 \pm 0.30^{a} \\
(100)\end{array}$ & $\begin{array}{c}18.83 \pm 0.29^{a} \\
(100)\end{array}$ & $18.50 \pm 0.29^{a}(75)$ & $\begin{array}{c}11.55 \pm 0.33^{a} \\
(100)\end{array}$ & $\begin{array}{c}20.65 \pm 0.26^{a} \\
(100)\end{array}$ \\
\hline $9 a$ & $11.80 \pm 0.25^{a}(75)$ & $22.74 \pm 0.32^{a}(50)$ & $\begin{array}{c}10.12 \pm 0.25^{a} \\
(100)\end{array}$ & $18.18 \pm 0.27^{a}(75)$ & $\begin{array}{c}15.37 \pm 0.31^{a} \\
(100)\end{array}$ \\
\hline $10 a$ & $18.72 \pm 0.35^{a}(75)$ & $\begin{array}{c}14.88 \pm 0.36^{a} \\
(100)\end{array}$ & $\begin{array}{c}18.20 \pm 0.32^{a} \\
(100)\end{array}$ & $19.88 \pm 0.34^{a}(75)$ & $22.31 \pm 0.30^{a}(50)$ \\
\hline $11 a$ & $\begin{array}{c}16.48 \pm 0.41^{a} \\
(100)\end{array}$ & $20.35 \pm 0.27^{a}(50)$ & $\begin{array}{c}13.29 \pm 0.52^{a} \\
(100)\end{array}$ & $9.33 \pm 0.45^{a}(100)$ & $20.02 \pm 0.38^{a}(75)$ \\
\hline $12 a$ & $20.84 \pm 0.31^{a}(75)$ & $15.38 \pm 0.30^{a}(75)$ & $23.13 \pm 0.30^{a}(50)$ & $16.92 \pm 0.41^{a}(50)$ & $\begin{array}{c}26.70 \pm 0.34^{a} \\
(100)\end{array}$ \\
\hline $13 a$ & $26.84 \pm 0.40^{\circ}(25)$ & $23.68 \pm 0.42^{a}(50)$ & $26.45 \pm 0.31^{a}(50)$ & $5.09 \pm 0.43^{a}(100)$ & $28.21 \pm 0.33^{a}(50)$ \\
\hline $14 a$ & $26.62 \pm 0.33^{c}(50)$ & $22.42 \pm 0.33^{a}(50)$ & $26.49 \pm 0.29^{a}(50)$ & $23.15 \pm 0.34^{a}(50)$ & $27.26 \pm 0.35^{a}(50)$ \\
\hline $15 a$ & $\begin{array}{c}14.60 \pm 0.31^{c} \\
(100)\end{array}$ & $\begin{array}{c}12.78 \pm 0.31^{a} \\
(100)\end{array}$ & $21.69 \pm 0.36^{a}(50)$ & $16.08 \pm 0.46^{a}(25)$ & $21.91 \pm 0.31^{a}(50)$ \\
\hline $16 a$ & $\begin{array}{c}15.80 \pm 0.29^{a} \\
(100)\end{array}$ & $19.23 \pm 0.31^{a}(75)$ & $17.73 \pm 0.40^{a}(75)$ & $24.25 \pm 0.32^{a}(25)$ & $15.34 \pm 0.26^{a}(75)$ \\
\hline $17 a$ & $\begin{array}{c}12.19 \pm 0.36^{a} \\
(100)\end{array}$ & $15.42 \pm 0.18^{a}(75)$ & $21.12 \pm 0.35^{a}(50)$ & $16.55 \pm 0.26^{a}(75)$ & $25.27 \pm 0.24^{a}(50)$ \\
\hline $18 a$ & $22.24 \pm 0.27^{a}(50)$ & $18.06 \pm 0.30^{a}(75)$ & $\begin{array}{c}12.79 \pm 0.56^{a} \\
(100)\end{array}$ & $16.80 \pm 0.40^{a}(75)$ & $23.52 \pm 0.43^{a}(50)$ \\
\hline Ofloxacin & $26.45 \pm 0.36^{a}(25)$ & $27.99 \pm 0.03^{a}(25)$ & $29.55 \pm 0.51^{a}(25)$ & $\begin{array}{c}30.66 \pm 0.41^{a} \\
(12.5)\end{array}$ & $\begin{array}{c}30.90 \pm 0.29^{a} \\
(12.5)\end{array}$ \\
\hline $\begin{array}{l}\text { Negative } \\
\text { Control }\end{array}$ & $0.0 \pm 0.0$ & $0.0 \pm 0.0$ & $0.0 \pm 0.0$ & $0.0 \pm 0.0$ & $0.0 \pm 0.0$ \\
\hline
\end{tabular}


Table 2: Antibacterial activity data of the title compounds (1a-18a) against Gram negative bacteria

\begin{tabular}{|c|c|c|c|c|c|}
\hline \multirow[t]{2}{*}{ Compound } & \multicolumn{5}{|c|}{ Zone of inhibition (mm \pm SD with corresponding MIC $(\mu \mathrm{g} / \mathrm{mL})$ in brackets } \\
\hline & E. coli & P. aeruginosa & K. pneumonia & B. bronchiseptica & P. vulgaris \\
\hline $1 \mathrm{a}$ & $16.60 \pm 0.30^{a}(100)$ & $13.92 \pm 0.54^{a}(75)$ & $15.90 \pm 0.45^{a}(100)$ & $24.10 \pm 0.47^{a}(50)$ & $20.84 \pm 0.54^{a}(50)$ \\
\hline $2 a$ & $11.64 \pm 0.29^{a}(100)$ & $17.44 \pm 0.42^{a}(75)$ & $10.16 \pm 0.45^{a}(100)$ & $18.88 \pm 0.35^{a}(75)$ & $16.86 \pm 0.45^{a}(100)$ \\
\hline 3a & $19.70 \pm 0.36^{a}(50)$ & $29.02 \pm 0.44^{a}(50)$ & $23.32 \pm 0.49^{a}(50)$ & $24.46 \pm 0.38^{a}(25)$ & $26.72 \pm 0.40^{a}(50)$ \\
\hline $4 a$ & $19.74 \pm 0.54^{a}(50)$ & $21.99 \pm 0.48^{a}(50)$ & $27.15 \pm 0.47^{a}(50)$ & $14.94 \pm 0.61^{a}(100)$ & $15.39 \pm 0.41^{a}(100)$ \\
\hline $5 a$ & $16.44 \pm 0.47^{a}(100)$ & $14.30 \pm 0.38^{a}(100)$ & $26.00 \pm 0.40^{a}(50)$ & $27.86 \pm 0.32^{a}(50)$ & $27.96 \pm 0.49^{a}(50)$ \\
\hline $6 a$ & $31.04 \pm 0.32^{a}(25)$ & $32.94 \pm 0.35^{a}(25)$ & $33.20 \pm 0.30^{a}(25)$ & $33.16 \pm 0.35^{a}(25)$ & $33.36 \pm 0.31^{a}(25)$ \\
\hline $7 a$ & $22.70 \pm 0.50^{a}(50)$ & $25.92 \pm 0.47^{a}(50)$ & $34.46 \pm 0.35^{c}(25)$ & $13.58 \pm 0.49^{a}(100)$ & $19.16 \pm 0.44^{a}(75)$ \\
\hline $8 a$ & $8.98 \pm 0.49^{a}(100)$ & $18.32 \pm 0.42^{a}(100)$ & $14.00 \pm 0.42^{a}(100)$ & $22.16 \pm 0.41^{a}(50)$ & $15.04 \pm 0.40^{a}(100)$ \\
\hline $9 a$ & $19.46 \pm 0.43^{a}(75)$ & $24.14 \pm 0.42^{a}(50)$ & $14.88 \pm 0.49^{a}(100)$ & $16.74 \pm 0.41^{a}(100)$ & $26.26 \pm 0.43^{a}(50)$ \\
\hline $10 \mathbf{a}$ & $25.50 \pm 0.36^{a}(50)$ & $33.04 \pm 0.44^{a}(50)$ & $27.84 \pm 0.42^{a}(50)$ & $28.86 \pm 0.36^{a}(50)$ & $10.54 \pm 0.46^{a}(100)$ \\
\hline $11 a$ & $28.83 \pm 0.34^{a}(25)$ & $25.82 \pm 0.32^{a}(50)$ & $24.30 \pm 0.46^{a}(50)$ & $20.64 \pm 0.47^{a}(50)$ & $29.10 \pm 0.46^{a}(25)$ \\
\hline $12 a$ & $19.70 \pm 0.50^{a}(75)$ & $23.68 \pm 0.26^{a}(50)$ & $26.08 \pm 0.52^{a}(50)$ & $28.54 \pm 0.46^{a}(50)$ & $15.34 \pm 0.32^{a}(100)$ \\
\hline $13 \mathbf{a}$ & $15.66 \pm 0.34^{a}(100)$ & $17.66 \pm 0.32^{a}(75)$ & $23.64 \pm 0.31^{a}(50)$ & $20.00 \pm 0.35^{a}(50)$ & $16.88 \pm 0.32^{a}(100)$ \\
\hline $14 a$ & $25.76 \pm 0.42^{a}(50)$ & $24.56 \pm 0.37^{a}(50)$ & $31.68 \pm 0.47^{a}(50)$ & $30.62 \pm 0.36^{a}(25)$ & $19.70 \pm 0.30^{a}(100)$ \\
\hline $15 \mathbf{a}$ & $14.76 \pm 0.48^{a}(100)$ & $11.24 \pm 0.49^{a}(100)$ & $13.50 \pm 0.45^{a}(175)$ & $21.92 \pm 0.45^{a}(75)$ & $16.59 \pm 0.30^{a}(100)$ \\
\hline $16 a$ & $27.30 \pm 0.50^{a}(25)$ & $31.64 \pm 0.44^{a}(25)$ & $20.38 \pm 0.38^{a}(75)$ & $29.18 \pm 0.52^{a}(50)$ & $27.54 \pm 0.43^{a}(50)$ \\
\hline $17 a$ & $17.54 \pm 0.33^{a}(75)$ & $25.95 \pm 0.45^{a}(50)$ & $20.37 \pm 0.33^{a}(50)$ & $29.82 \pm 0.20^{a}(50)$ & $25.44 \pm 0.37^{a}(50)$ \\
\hline $18 a$ & $24.14 \pm 0.41^{a}(50)$ & $29.72 \pm 0.37^{a}(50)$ & $25.77 \pm 0.35^{a}(50)$ & $28.92 \pm 0.44^{a}(50)$ & $22.89 \pm 0.39^{a}(50)$ \\
\hline Ofloxacin & $30.32 \pm 0.41^{a}(12.5)$ & $32.23 \pm 0.15^{a}(12.5)$ & $32.70 \pm 0.21^{a}(12.5)$ & $33.44 \pm 0.22^{a}(25)$ & $30.97 \pm 0.32^{a}(12.5)$ \\
\hline $\begin{array}{l}\text { Negative } \\
\text { Control }\end{array}$ & & & $0.0 \pm 0.0$ & $0.0 \pm 0.0$ & \\
\hline
\end{tabular}

Values in parenthesis represent the corresponding MIC $(\mu \mathrm{g} / \mathrm{mL}) ;{ }^{a} p<0.0001,{ }^{b} p<0.001,{ }^{c} p<0.05$

Table 3: Antifungal activity data of the title compounds (1a-18a) against fungi

\begin{tabular}{|c|c|c|c|c|c|}
\hline \multirow[t]{2}{*}{ Compound } & \multicolumn{5}{|c|}{ Zone of inhibition ( $\mathrm{mm} \pm$ SD with corresponding MIC $(\mu \mathrm{g} / \mathrm{mL})$ in brackets } \\
\hline & C. albicans & A. niger & A. flavus & M. purpureous & P. citrinum \\
\hline 1a & $31.85 \pm 0.37^{a}(25)$ & $35.14 \pm 0.29^{C}(25)$ & $34.97 \pm 0.37^{d}(25)$ & $28.64 \pm 0.45^{a}(25)$ & $23.53 \pm 0.43^{a}(50)$ \\
\hline $2 a$ & $29.35 \pm 0.35^{a}(50)$ & $35.46 \pm 0.27^{d}(25)$ & $31.48 \pm 0.32^{c}(25)$ & $25.48 \pm 0.46^{a}(50)$ & $26.41 \pm 0.49^{a}(50)$ \\
\hline 3a & $23.75 \pm 0.37^{a}(50)$ & $23.18 \pm 0.51^{a}(50)$ & $27.52 \pm 0.37^{a}(50)$ & $31.50 \pm 0.27^{a}(25)$ & $20.51 \pm 0.31^{a}(50)$ \\
\hline $4 a$ & $24.54 \pm 0.36^{a}(50)$ & $27.97 \pm 0.34^{a}(25)$ & $22.49 \pm 0.25^{a}(50)$ & $19.56 \pm 0.42^{a}(50)$ & $26.49 \pm 0.45^{a}(25)$ \\
\hline $5 a$ & $29.60 \pm 0.43^{a}(50)$ & $18.85 \pm 0.37^{a}(50)$ & $25.28 \pm 0.44^{a}(50)$ & $23.96 \pm 0.38^{a}(50)$ & $24.93 \pm 0.44^{a}(50)$ \\
\hline $6 a$ & $34.20 \pm 0.39^{a}(25)$ & $28.53 \pm 0.43^{a}(25)$ & $33.67 \pm 0.36^{a}(25)$ & $31.81 \pm 0.39^{a}(25)$ & $36.57 \pm 0.32^{a}(25)$ \\
\hline $7 a$ & $\begin{array}{c}25.18 \pm 0.484^{a} \\
(50)\end{array}$ & $26.36 \pm 0.44^{a}(50)$ & $22.79 \pm 0.38^{a}(50)$ & $23.75 \pm 0.42^{a}(50)$ & $27.99 \pm 0.51^{a}(25)$ \\
\hline $8 \mathbf{a}$ & $28.86 \pm 0.38^{a}(50)$ & $24.44 \pm 0.55^{a}(50)$ & $22.53 \pm 0.52^{a}(50)$ & $29.12 \pm 0.45^{a}(25)$ & $25.06 \pm 0.37^{a}(50)$ \\
\hline $9 a$ & $21.64 \pm 0.44^{a}(50)$ & $30.09 \pm 0.33^{a}(25)$ & $23.68 \pm 0.42^{a}(25)$ & $18.29 \pm 0.42^{a}(50)$ & $30.96 \pm 0.29^{a}(25)$ \\
\hline $10 a$ & $18.37 \pm 0.31^{a}(50)$ & $25.71 \pm 0.47^{a}(50)$ & $26.36 \pm 0.32^{b}(50)$ & $24.67 \pm 0.35^{a}(50)$ & $28.08 \pm 0.35^{a}(25)$ \\
\hline $11 a$ & $33.28 \pm 0.35^{a}(25)$ & $28.88 \pm 0.38^{a}(25)$ & $26.69 \pm 0.48^{a}(25)$ & $34.99 \pm 0.31^{a}(25)$ & $36.62 \pm 0.29^{b}$ \\
\hline $12 a$ & $22.42 \pm 0.42^{a}(50)$ & $31.74 \pm 0.44^{a}(25)$ & $23.36 \pm 3.39^{a}(25)$ & $22.06 \pm 0.52^{a}(50)$ & $21.67 \pm 0.45^{a}(50)$ \\
\hline $13 a$ & $32.86 \pm 0.41^{a}(25)$ & $35.75 \pm 0.22^{d}(25)$ & $33.19 \pm 0.33^{a}(25)$ & $27.65 \pm 0.38^{a}(50)$ & $25.83 \pm 0.37^{a}(50)$ \\
\hline $14 a$ & $31.42 \pm 0.41^{a}(25)$ & $35.20 \pm 0.33^{c}(25)$ & $26.58 \pm 0.55^{a}(25)$ & $36.01 \pm 0.17^{a}(25)$ & $30.35 \pm 0.42^{a}(25)$ \\
\hline $15 a$ & $26.87 \pm 0.57^{a}(50)$ & $21.99 \pm 0.60^{a}(50)$ & $30.09 \pm 0.37^{a}(25)$ & $26.39 \pm 0.38^{a}(50)$ & $28.76 \pm 0.35^{c}(25)$ \\
\hline $16 a$ & $30.13 \pm 0.37^{a}(25)$ & $29.29 \pm 0.39^{a}(25)$ & $34.06 \pm 0.29^{a}(25)$ & $35.23 \pm 0.47^{a}(25)$ & $32.33 \pm 0.66^{a}(25)$ \\
\hline $17 a$ & $36.11 \pm 0.48^{a}(25)$ & $34.21 \pm 0.43^{a}(25)$ & $32.30 \pm 0.49^{a}(25)$ & $36.18 \pm 0.35^{a}(25)$ & $35.33 \pm 0.31^{a}(25)$ \\
\hline $18 a$ & $32.00 \pm 0.49^{a}(25)$ & $29.55 \pm 0.46^{a}(25)$ & $34.61 \pm 0.47^{d}(25)$ & $26.97 \pm 0.39^{a}(50)$ & $31.61 \pm 0.36^{c}(25)$ \\
\hline Ketoconazole & $\begin{array}{c}33.30 \pm 0.31^{a} \\
(12.5)\end{array}$ & $\begin{array}{c}26.99 \pm 0.44^{a} \\
(12.5)\end{array}$ & $29.51 \pm 0.22^{a}(25)$ & $30.58 \pm 0.12^{a}(12.5)$ & $27.11 \pm 0.31^{a}(25)$ \\
\hline $\begin{array}{l}\text { Negative } \\
\text { Control }\end{array}$ & $0.0 \pm 0.0$ & $0.0 \pm 0.0$ & $0.0 \pm 0.0$ & $0.0 \pm 0.0$ & $0.0 \pm 0.0$ \\
\hline
\end{tabular}

Values in parenthesis represent the corresponding MIC $(\mu \mathrm{g} / \mathrm{mL}) ;{ }^{a} p<0.0001,{ }^{b} p<0.001,{ }^{c} p<0.05$

The compounds (1a-18a) were tested for their in vitro antimicrobial activity by serial plate dilution method [26,27] against five Gram positive bacteria; five Gram-negative bacteria; and five fungi. The compound $\mathbf{6 a}$ (MIC $=50 \mu \mathrm{g} / \mathrm{mL} ; p<$ 0.05 or less) displayed highest activity against $S$. aureus, E. faecalis, S. epidermidis, B. subtilis, and $B$. cereus. The compound 6 a $(\mathrm{MIC}=25$ $\mu \mathrm{g} / \mathrm{mL} ; p<0.05$ or less) further displayed very good activity against E. coli; $P$. aeruginosa $K$. pneumonia, $B$. bronchiseptica, and $P$. vulgaris, respectively. Compounds $6 \mathrm{a}(\mathrm{MIC}=25 \mu \mathrm{g} / \mathrm{mL} ; p$ $<0.0001)$ and 17a (MIC = $25 \mu \mathrm{g} / \mathrm{mL} ; \mathrm{p}<0.0001)$ showed very good activity against $C$. albicans, $A$. niger, $A$. flavus, $M$. purpureous, and $P$. citrinum, respectively. 
It is evident from the antimicrobial activity data mentioned in Table 1, Table 2, and Table 3 that the title compounds are better antifungal agents than antibacterial agents. These results also support our earlier hypothesis [25] that the replacement of the morpholine moiety by its bioisosteres like piperidine moiety in these type of compounds may produce promising antifungal compounds. It is also evident from the antimicrobial activity data that these piperidine ring containing derivative are more potent antimicrobial agents than the reported morpholine ring containing derivatives [25] or the free amino group containing similar compounds [24]. It is also believed that the synthesized compounds might be inhibiting the growth of all tested microorganism by same mechanism as earlier reported pyrimidine moiety containing drugs [15].

The structure activity relationship study of the title compounds (1a-18a) revealed that replacement of the morpholine ring [25] by the piperidine ring increases the overall antimicrobial activity of these type of compounds, predominantly the antifungal activity. The bromo group at 7-postion of the coumarin moiety along with the 4-chlorophenyl group at position-6 of the pyrimidine ring provides the most promising antimicrobial agent (6a) that is effective against Gram positive, Gram negative and fungi. The replacement of the 7-bromo group with the 7chloro group, and presence of an additional chloro group at 4-cholorophenyl ring present at postion- 6 of pyrimidine ring provides compound (17a) having promising antifungal activity. This structure activity relationship also suggests that the free amino group of the reported compounds [24] may further be exploited for the identification of more potent and safe antimicrobial agents.

\section{CONCLUSION}

It is evident from the antimicrobial activity data of the title compounds (1a-18a) that the compounds $6 a$ and $17 a$ are the promising antimicrobial agents of this series of compounds. However, these compounds produced promising effect at higher concentration, and therefore, are still considered to be less potent than standard drugs, ofloxacin and ketoconazole. There is a possibility that the replacement of the piperidine moiety by other similar type of chemical moieties, for example, pyrrolidine moiety may produce promising potent antimicrobial agents that are effective against Gram positive bacteria, Gram negative bacteria and fungi. Accordingly, this study may be extended to acquire more information about the structure activity relationships of this series of compounds.

\section{DECLARATIONS}

\section{Acknowledgement}

The authors are thankful to Central Drug Research Institute (CDRI) for generating the spectral data of the title compounds.

\section{Conflict of Interest}

No conflict of interest associated with this work.

\section{Contribution of Authors}

The authors declare that this work was done by the authors named in this article and all liabilities pertaining to claims relating to the content of this article will be borne by them.

\section{REFERENCES}

1. Abida, Imran M, Hagga MAM, El-Feky SA. New chemical entities of future for infectious diseases. Eur J Exp Biol 2014; 4(1): 613-624.

2. Jain KS, Chitre TS, Miniyar PB, Kathiravan MK, Bendre VS, Veer VS, Shahane SR, Shishoo CJ. Biological and medicinal significance of pyrimidines. Curr Sci 2006; 90(6): 793-703.

3. Jitendra KG, Pramod KS, Rupesh D, Anshu C, Avnesh S, Verma PK, Sambhu CM, Rakesh KY, Shivjee $K$. Analgesic study of novel pyrimidine derivatives linked with coumarin moiety. Med Chem Res 2012; 21(8): 1625-1632.

4. Anshu C, Pramod KS, Prabhakar V, Nitin K, Rupesh D. Microwave assisted synthesis of novel pyrimidine derivatives and investigation of their analgesic and ulcerogenic activity. Med Chem Res 2012; 21(11): 36293645.

5. Kumar SM, Pavani M, Bhalgat CM, Deepthi R, Mounika A, Mudshinge SR, Reas IJ, Ghomi JS, Ghasemzadeh $M A$. Novel pyrimidine and its triazole fused derivatives: synthesis and investigation of antioxidant and antiinflammatory activity. J Serb Chem Soc 2011; 76: 679684.

6. Abu-Hashem AA, Youssef MM, Hoda AR. Synthesis, antioxidant, antitumor activities of some new thiazolopyrimidines, pyrrolothiazolopyrimidines and triazolopyrrolo thiazolopyrimidines derivatives. J Chi Chem Soc 2011; 58: 41-48.

7. Gressler V, Moura S, Flores AFC, Flores DC, Colepicolo $P$, Pinto E. Antioxidant and antimicrobial properties of 2(4,5-dihydro-1H-pyrazol-1-yl)-pyrimidine and 1carboxamidino-1H-pyrazole derivatives. J Braz Chem Soc 2010; 21: 1-7. 
8. Aggarwal R, Masan E, Kaushik P, Kaushik D, Sharma C, Aneja KR. Synthesis and biological evaluation of 7trifluoromethylpyrazolo\{1,5-a\}pyrimidines as antiinflammatory and antimicrobial agents. J Fluorine Chem 2014; 168: 16-24.

9. Kumar N, Chauhan A, Drabu S. Synthesis of cyanopyridine and pyrimidine analogues as new antiinflammatory and antimicrobial agents. Biomed Pharmacother 2011; 65(5): 375-380.

10. Mohamed MS, Youns MM, Ahmed NM. Novel indolylpyrimidine derivatives: synthesis, antimicrobial, and antioxidant evaluations. Med Chem Res 2014; 23(7): 3374-3388.

11. Kotaiah Y, Nagaraju K, Harikrishna N, Rao CV, Yamini L, Vijjulatha M. Synthesis, docking and evaluation of antioxidant and antimicrobial activities of novel 1,2,4triazolo\{3,4-b\}\{1,3,4\}thiadiazol-6-yl)selenopheno\{2,3d\}pyrimidines. Eur J Med Chem 2014; 75: 195-102.

12. Mosaad SM, Mahmoud MY, Naglaa MA. Novel indolylpyrimidine derivatives: synthesis, antimicrobial, and antioxidant evaluations. Med Chem Res 2014; 23: 3374 3388.

13. Saundane AR, Yarlakatti M, Kalpana R. Synthesis, antimicrobial, and antioxidant activities of some new indole analogues containing pyrimidine and fused pyrimidine systems. Med Chem Res 2012; 21: 38093817.

14. Selvam TP, James CR, Dniandev PV, Valzita SK. A mini review of pyrimidine and fused pyrimidine marketed Drugs. Res Pharm 2012; 2(4): 1-9.

15. Sharma V, Chitranshi N, Agarwal AK. Significance and biological importance of pyrimidine in the microbial world. Int J Med Chem 2014; doi:10.1155/2014/202784.

16. Patil B, Banerjee S. Importance of Piperidine Moiety in Medicinal Chemistry Research: A Review. J Pharm Res 2012; 5(12): 5493-5509.

17. Maruthavanan T, Venkatesan P. Synthesis of 4-hydroxy3,4-dialkyl-2,6-diaryl-piperidine derivatives as potent antimicrobial agent. Nat Prod Res 2013; 27(3): 238-245.

18. Siwek A, Stefańska J. Antimicrobial activity and SAR study of some novel thiosemicarbazide derivatives bearing piperidine moiety. Med Chem 2011; 7(6): 690696.

19. Vinaya K, Kavitha $R$, Ananda KCS, Benaka PSB, Chandrappa S, Deepak SA, Nanjunda SS, Umesha S, Rangappa KS. Synthesis and antimicrobial activity of 1benzhydryl-sulfonyl-4-(3-(piperidin-4-yl) propyl) piperidine derivatives against pathogens of Lycopersicon esculentum: a structure-activity evaluation study. Arch Pharm Res 2009; 32(1): 33-41.

20. Arslan S, Logoglu E, Oktemer A. Antimicrobial activity studies on some piperidine and pyrrolidine substituted halogenobenzene derivatives. J Enzyme Inhib Med Chem 2006; 21(2): 211-214.

21. Imran M, Khan SA. Synthesis of novel 3,5-disubstituted isoxazolines as potential antibacterial and antifungal agents. Indian J Heterocycl Chem 2004; 13: 213-216.

22. Khan SA, Siddiqui N, Imran M, Haque SW. Synthesis and antimicrobial screening of novel mannich bases of isatin derivative. Indian J Pharm Sci 2005; 66(6): 830-834.

23. Imran M, Abida, Khan SA. Synthesis and Antimicrobial Activity of Some 2-Amino-4-(7-Substituted/Unsubstituted Coumarin-3-yl)-6-(Chlorosubstitutedphenyl) Pyrimidines. Trop J Pharm Res 2015; 14(7): 1265-1272.

24. Abida, Alaqel S, Imran M, El-Feky SA, Khan SA. Synthesis and antimicrobial activity of 3(substitutedphenyl)-1-(7-substitutedcoumarin-3-yl)prop2-ene-1-ones. J Chem Pharm Res 2013; 5(12): 10891093.

25. Imran M, Abida, Alsalman AJ. Synthesis and evaluation of antimicrobial activity of some 2morpholinomethylamino-4-(7-unsubstituted/ substitutedcoumarin-3-yl)-6-chlorosubstituted phenyl Pyrimidines. Trop J Pharm Res 2016; 15(2): 393-404.

26. Barry AL. Procedures and theoretical considerations for testing antimicrobial agents in agar media. In: Lorian $\mathrm{V}$, Ed. Antibiotics in Laboratory Medicine, 3rd edn. Baltimore, Williams \& Wilkins; 1991; pp 1-16.

27. Varma RS, Khan ZK, Singh AP. Eds. Antifungal agents: past, present, future prospects. Lucknow: National Academy of Chemistry and Biology, India, 1998; pp 55128. 